Check for updates

Cite this: RSC Adv., 2017, 7, 42180

Received 22nd June 2017

Accepted 24th August 2017

DOI: 10.1039/c7ra06951k

rsc.li/rsc-advances

\section{Mechanofluorochromic properties of fluorescent molecules based on a dicyanomethylene-4H-pyran and indole isomer containing different alkyl chains via an alkene module $\uparrow$}

\author{
Lebin Qian, ${ }^{a}$ Yibin Zhou, ${ }^{a}$ Miaochang Liu, ${ }^{a}$ Xiaobo Huang, (D) *a Ge Wu, (D) b \\ Wenxia Gao, ${ }^{a}$ Jinchang Ding ${ }^{a}$ and Huayue $\mathrm{Wu}^{\star a}$
}

A series of 3-, 4-, and 5-position indole-substituted dicyanomethylene-4H-pyran (I3PM, I4PM, and I5PM) derivatives with different alkyl chains were synthesized and their mechanofluorochromic (MFC) properties were investigated. Compared with the alkyl chains, the isomerization of the indole unit plays a more important role in regulating the MFC properties of these compounds. The increase of the alkyl chain length and the isomerization of the alkyl chain are favorable to the effective mechanofluorochromism of the I3PM derivatives, however, the former exhibits the opposite effect on that of the I4PM derivatives. The opposite alkyl length-dependent MFC behavior is closely related to the degree of distortion of the molecular conformation, namely the more twisted the molecular conformation, the more pronounced the MFC phenomenon. In particular, the introduction of the alkyl chains is completely detrimental to the MFC properties of the I5PM derivatives. X-Ray diffraction experiments indicate that the MFC properties of some of these compounds should be mainly attributed to the transformation from a crystalline state to an amorphous state because the weak intermolecular interactions and loose molecular packing modes in their solid-state samples can be easily damaged under external force stimuli as revealed by the single crystal $\mathrm{X}$-ray diffraction analysis.

\section{Introduction}

Mechanofluorochromic (MFC) organic molecules are a class of "smart" materials whose solid-state fluorescence colors can display obvious changes in response to external force stimuli, and have received considerable interest owing to their potential applications in the fields of fluorescent sensors, ${ }^{1}$ data storage, ${ }^{2}$ security inks, ${ }^{3}$ optoelectronic devices, ${ }^{4}$ etc. In general, to make an organic fluorescence molecule have MFC properties, first the molecule must be capable of emitting fluorescence in the solid state, and its solid morphology must be able to undergo a transition from a crystalline to an amorphous state or from a crystalline state to another crystalline state under pressure stimuli. ${ }^{5}$ Although the development new fluorescent molecular structures and the structural modification of traditional fluorescent molecular frameworks to meet the above two conditions are both crucial for obtaining new MFC materials, the latter

${ }^{a}$ College of Chemistry and Materials Engineering, Wenzhou University, Wenzhou, 325035, P. R. China.E-mail: xiaobhuang@wzu.edu.cn; huayuewu@wzu.edu.cn ${ }^{b}$ School of Pharmacy, Wenzhou Medical University, Wenzhou 325035, P. R. China $\dagger$ Electronic supplementary information (ESI) available: UV-vis spectra, fluorescence spectra, photophysical data, XRD curves, and other additional information. CCDC 1554482-1554487. For ESI and crystallographic data in CIF or other electronic format see DOI: 10.1039/c7ra06951k looks more alluring and more easily accepted by researchers. Generally speaking, the introduction of alkyl chains to the conjugated skeleton of organic fluorescence molecules is to mainly tune the solubility of the materials, but some recent studies show that alkyl chains can also influence the intermolecular interactions and molecular packing modes of the materials in the solid state, and thereby play a crucial role in tuning and altering their MFC properties. ${ }^{6}$ Although the change of the length of alkyl chain can greatly affect MFC behaviors of some fluorescent molecules, this effect is very complicated. For instance, longer alkyl chains are favorable to the more remarkable MFC phenomena of 9,10-bis[(9,9-dialkylfluorene-2yl)vinyl-2] anthracenes, ${ }^{7}$ 9,10-bis( $N$-alkylphenothiazin-3-yl-vinyl2)anthracenes, ${ }^{8} \quad$ and $\quad 9,10$-bis ( $p$-alkoxystyryl)anthracenes, ${ }^{9}$ whereas obviously disadvantageous to those of 9,10-bis[( $N$-alkylcarbazol-3-yl)-vinyl-2]anthracenes ${ }^{10}$ and 9,10-bis( $N$-alkylindole3-yl-vinyl-2)anthracenes, ${ }^{\mathbf{1 1}}$ and 2,5-dialkylcarbazole-substituted terephthalate derivatives. ${ }^{12}$ Moreover, the branched alkyl chains are reported to be advantageous to obtain high-contrast MFC compounds for 9,10-bis(butoxystyryl)anthracenes. ${ }^{13}$ Additionally, some other ways have also proven to be very convenient in regulating the MFC properties of the fluorescent molecules, such as the introduction of appropriate aromatic substituents ${ }^{\mathbf{1 4}}$ and heavy atoms ${ }^{15}$ as well as changing the position of the 
substituent. ${ }^{16}$ But so far the influence of the structure of the molecule on its MFC property is still not very clear and the regular pattern is hard to sum up, which is worthy of further study for the development of MFC-active materials based on the traditional fluorescent molecular frameworks.

$4 H$-Pyran is a traditional and excellent building unit for constructing donor- $\pi$-acceptor $(\mathrm{D}-\pi-\mathrm{A})$ fluorescent compounds in the applications of organic electroluminescent diodes, molecular logic gates, and fluorescent sensors. ${ }^{\mathbf{1 7}}$ However, MFC materials based on $4 H$-pyran unit have rarely been reported until now. Very recently, we found that some indene-1,3-dionemethylene- $4 H$-pyran derivatives containing different lengths of alkoxy groups showed obvious alkoxy length-dependent MFC properties and the longer alkoxy chain led to more remarkable MFC phenomena. ${ }^{18}$ Moreover, for naphthylethylene-substituted dicyanomethylene- $4 H$-pyran derivatives and cyano(ethoxycarbonyl)methylene- $4 H$-pyran derivatives, the position isomerism of the naphthylethylene end groups were found to significantly affect their solid-state fluorescence color changes under external stimuli. ${ }^{19}$ To our knowledge, the combined effects of two different factors, namely different alkyl groups and the isomerization of aromatic substituents on the MFC properties of the fluorescent molecules has not been reported. Herein, a series of indolesubstituted dicyanomethylene- $4 H$-pyran (IPM) derivatives with different alkyl chains and varying the linking position of the indole from 3-, 4- to 5-position at $4 H$-pyran ring, namely I3PM, I4PM, and I5PM, were synthesized. The combined effects of isomerization and length of the alkyl chains and position isomerization of the indole unit on the MFC properties of the target compounds were systematically investigated. The results show the isomerization of indole unit plays a more important role in regulating the MFC properties of these compounds compared with the alkyl chains. The isomerization and length of the alkyl chains exhibit different effects on the MFC properties of these fluorescent molecules when the substitution position of the indole unit is different. The increase of the alkyl chain length and the isomerization of the alkyl chain are favorable to the effective MFC properties of the I3PM derivatives, however, the former exhibits opposite effects on those of the I4PM derivatives. In particular, the introduction of the alkyl chains is completely detrimental to the MFC properties of the I5PM derivatives. The results signify that the choice of the alkyl chains and the isomerization of indole unit can be used to regulate the MFC properties of the IPM derivatives. X-Ray diffraction (XRD) experiments reveal that the reversible MFC properties of the IPM derivatives should be mainly attributed to the transformation between amorphous and crystalline states.

\section{Experimental}

\section{Measurements and materials}

NMR spectral measurements were performed on a Bruker DRX 500 NMR spectrometer. Mass spectrometry spectra were performed with a Finnigan DECAX-30000 LCQ Deca mass spectrometer or a Finngan MAT95XP mass spectrometer. Elemental analysis was recorded on an Elementar Vario MICRO analyzer.
Fluorescence measurements were carried out with a HITACHI F7000 fluorometer. X-Ray diffraction (XRD) measurements were carried out with a Bruker D8 Advance X-ray diffractometer. Single-crystal X-ray diffraction measurements were carried out with a Bruker-Nonius Smart Apex CCD diffractometer with graphite monochromated Mo $\mathrm{K} \alpha$ radiation. 2,6-Dimethyl-4pyrone (1), malononitrile, $1 \mathrm{H}$-indole-3-carbaldehyde (3a), $1 \mathrm{H}^{-}$ indole-4-carbaldehyde (4a), $1 H$-indole-5-carbaldehyde (5a), and various alkyl bromides were purchased from commercial suppliers and used directly.

\section{Synthesis of $N$-substituted indolecarboxaldehydes $3 b-3 d / 4 b-$ $4 d / 5 b-5 d$}

General procedure: Alkyl bromide $(20.0 \mathrm{mmol})$ was slowly added to a solution of $\mathbf{3 a} / \mathbf{4 a} / \mathbf{5 a}(2.47 \mathrm{~g}, 17.0 \mathrm{mmol})$ and $\mathrm{KOH}$ $(1.43 \mathrm{~g}, 25.60 \mathrm{mmol})$ in DMSO $(30 \mathrm{~mL})$. The mixture was stirred overnight at room temperature, and then added water and extracted with dichloromethane. The combined organic layers were washed with water and brine, dried over anhydrous $\mathrm{Na}_{2} \mathrm{SO}_{4}$, and evaporated to dryness in vacuo. The residue was purified by column chromatography through silica gel with petroleum ether/ethyl acetate $(\mathrm{v} / \mathrm{v}=4 / 1)$ to give the pure $N$ substituted indolecarboxaldehyde. The characterization data for the intermediates $\mathbf{3 b} \mathbf{- 3} \mathbf{3} / \mathbf{4} \mathbf{b}-\mathbf{4} \mathbf{d} / \mathbf{5} \mathbf{b}-\mathbf{5 d}$ are listed as follows:

1-Butyl-1H-indole-3-carbaldehyde (3b). Yellow liquid (3.29 g), 95.9\% yield. ${ }^{1} \mathrm{H} \mathrm{NMR}\left(\mathrm{CDCl}_{3}, 500 \mathrm{MHz}\right): \delta 9.95(\mathrm{~s}, 1 \mathrm{H}), 8.31$ (dd, $\left.{ }^{3} J=6.5 \mathrm{~Hz},{ }^{4} J=2.5 \mathrm{~Hz}, 1 \mathrm{H}\right), 7.65$ (s, 1H), 7.36-7.27 (m, 3H), 4.09 $(\mathrm{t}, J=7.5 \mathrm{~Hz}, 2 \mathrm{H}), 1.84-1.79(\mathrm{~m}, 2 \mathrm{H}), 1.35-1.31(\mathrm{~m}, 2 \mathrm{H}), 0.93(\mathrm{t}, J$ $=7.5 \mathrm{~Hz}, 3 \mathrm{H}) \mathrm{ppm} .{ }^{13} \mathrm{C} \mathrm{NMR}\left(\mathrm{CDCl}_{3}, 125 \mathrm{MHz}\right): \delta 184.2,138.2$, 137.1, 125.2, 123.6, 122.6, 121.8, 117.8, 110.0, 46.7, 31.5, 19.8, 13.4 ppm. Anal. calcd for $\mathrm{C}_{13} \mathrm{H}_{15} \mathrm{NO}$ : C, 77.58; H, 7.51; N, 6.96. Found: C, 77.99; H, 7.56; N, 6.91.

1-Isobutyl-1H-indole-3-carbaldehyde (3c). Yellow liquid $(3.20$ g), 93.3\% yield. ${ }^{1} \mathrm{H}$ NMR $\left(\mathrm{CDCl}_{3}, 500 \mathrm{MHz}\right): \delta 9.96(\mathrm{~s}, 1 \mathrm{H}), 8.29$ $\left(\mathrm{dd},{ }^{3} J=6.5 \mathrm{~Hz},{ }^{4} J=3.0 \mathrm{~Hz}, 1 \mathrm{H}\right), 7.64(\mathrm{~s}, 1 \mathrm{H}), 7.34-7.26(\mathrm{~m}, 3 \mathrm{H})$, 3.91 (d, $J=7.5 \mathrm{~Hz}, 2 \mathrm{H}), 2.24-2.16(\mathrm{~m}, 1 \mathrm{H}), 0.92$ (d, $J=6.5 \mathrm{~Hz}$, $6 \mathrm{H}) \mathrm{ppm} .{ }^{13} \mathrm{C} \mathrm{NMR}\left(\mathrm{CDCl}_{3}, 125 \mathrm{MHz}\right): \delta$ 184.4, 138.8, 137.4, 125.3, 123.7, 122.7, 121.9, 117.8, 110.2, 54.7, 29.0, 20.0 ppm. Anal. calcd for $\mathrm{C}_{13} \mathrm{H}_{15} \mathrm{NO}$ : C, 77.58; H, 7.51; N, 6.96. Found: C, 78.02; H, 7.55; N, 7.02.

1-Dodecyl-1H-indole-3-carbaldehyde (3d). White solid (4.93 g), 92.3\% yield. ${ }^{1} \mathrm{H}$ NMR $\left(\mathrm{CDCl}_{3}, 500 \mathrm{MHz}\right): \delta 10.00(\mathrm{~s}, 1 \mathrm{H}), 8.31$ $\left(\mathrm{dd},{ }^{3} J=6.5 \mathrm{~Hz},{ }^{4} J=2.0 \mathrm{~Hz}, 1 \mathrm{H}\right), 7.70(\mathrm{~s}, 1 \mathrm{H}), 7.38-7.29(\mathrm{~m}, 3 \mathrm{H})$, $4.15(\mathrm{t}, J=7.0 \mathrm{~Hz}, 2 \mathrm{H}), 1.90-1.87(\mathrm{~m}, 2 \mathrm{H}), 1.33-1.25(\mathrm{~m}, 18 \mathrm{H})$, $0.88(\mathrm{t}, J=7.0 \mathrm{~Hz}, 3 \mathrm{H}) \mathrm{ppm} .{ }^{13} \mathrm{C} \mathrm{NMR}\left(\mathrm{CDCl}_{3}, 125 \mathrm{MHz}\right): \delta 184.3$, 138.1, 137.2, 125.5, 123.8, 122.8, 122.1, 118.0, 110.0, 47.3, 31.9, 29.7, 29.53, 29.46, 29.37, 29.27, 29.1, 26.8, 22.6, 14.0 ppm. Anal. calcd for $\mathrm{C}_{21} \mathrm{H}_{31} \mathrm{NO}$ : C, 80.46; H, 9.97; N, 4.47. Found: C, 80.92; $\mathrm{H}, 9.93$; N, 4.44.

1-Butyl-1H-indole-4-carbaldehyde (4b). Dark yellow liquid (2.56 g), 74.6\% yield. ${ }^{1} \mathrm{H}$ NMR $\left(\mathrm{CDCl}_{3}, 500 \mathrm{MHz}\right): \delta 10.25(\mathrm{~s}, 1 \mathrm{H})$, $7.62(\mathrm{~d}, J=7.5 \mathrm{~Hz}, 2 \mathrm{H}), 7.35-7.29(\mathrm{~m}, 3 \mathrm{H}), 4.17(\mathrm{t}, J=7.0 \mathrm{~Hz}$, $2 \mathrm{H}), 1.85-1.79(\mathrm{~m}, 2 \mathrm{H}), 1.35-1.30(\mathrm{~m}, 2 \mathrm{H}), 0.94(\mathrm{t}, J=7.5 \mathrm{~Hz}$, $3 \mathrm{H})$ ppm. ${ }^{13} \mathrm{C} \mathrm{NMR}\left(\mathrm{CDCl}_{3}, 125 \mathrm{MHz}\right): \delta$ 192.9, 136.6, 131.2, $128.4,126.9$, 126.3, 120.6, 115.6, 101.3, 46.1, 32.4, 20.0, 
13.5 ppm. Anal. calcd for $\mathrm{C}_{13} \mathrm{H}_{15} \mathrm{NO}: \mathrm{C}, 77.58 ; \mathrm{H}, 7.51 ; \mathrm{N}, 6.96$. Found: C, 77.29; H, 7.47; N, 6.92.

1-Isobutyl-1H-indole-4-carbaldehyde (4c). Dark yellow liquid $(2.34 \mathrm{~g}), 68.2 \%$ yield. ${ }^{1} \mathrm{H} \mathrm{NMR}\left(\mathrm{CDCl}_{3}, 500 \mathrm{MHz}\right): \delta 10.24(\mathrm{~s}, 1 \mathrm{H})$, 7.59 (d, $J=7.5 \mathrm{~Hz}, 2 \mathrm{H}), 7.32-7.28(\mathrm{~m}, 3 \mathrm{H}), 3.94(\mathrm{~d}, J=7.5 \mathrm{~Hz}$, 2H), 2.21-2.13 (m, 1H), 0.90 (d, $J=7.0 \mathrm{~Hz}, 6 \mathrm{H}) \mathrm{ppm} .{ }^{13} \mathrm{C}$ NMR $\left(\mathrm{CDCl}_{3}, 125 \mathrm{MHz}\right): \delta$ 193.0, 137.0, 131.8, 128.4, 126.9, 126.2, 120.6, 115.8, 101.3, 54.1, 29.7, 20.1 ppm. Anal. calcd for $\mathrm{C}_{13} \mathrm{H}_{15} \mathrm{NO}$ : C, 77.58; H, 7.51; N, 6.96. Found: C, 77.18; H, 7.46; N, 6.90 .

1-Dodecyl-1H-indole-4-carbaldehyde (4d). Yellow liquid (3.86 g), 72.2\% yield. ${ }^{1} \mathrm{H}$ NMR $\left(\mathrm{CDCl}_{3}, 500 \mathrm{MHz}\right): \delta 10.25$ (s, $\left.1 \mathrm{H}\right), 7.62$ $(\mathrm{d}, J=7.5 \mathrm{~Hz}, 2 \mathrm{H}), 7.35-7.30(\mathrm{~m}, 3 \mathrm{H}), 4.16(\mathrm{t}, J=7.0 \mathrm{~Hz}, 2 \mathrm{H})$, 1.85-1.82 (m, 2H), 1.30-1.25 (m, 18H), $0.90(\mathrm{t}, J=7.5 \mathrm{~Hz}$, $3 \mathrm{H}) \mathrm{ppm} .{ }^{13} \mathrm{C} \mathrm{NMR}\left(\mathrm{CDCl}_{3}, 125 \mathrm{MHz}\right): \delta$ 192.9, 136.7, 131.2, 128.4, 126.9, 126.5, 120.6, 115.6, 101.4, 46.5, 31.8, 30.3, 29.52, $29.45,29.38,29.25,29.11,26.9,22.6,14.0$ ppm. Anal. calcd for $\mathrm{C}_{21} \mathrm{H}_{31} \mathrm{NO}$ : C, 80.46; H, 9.97; N, 4.47. Found: C, 81.05; H, 9.94; N, 4.49 .

1-Butyl-1H-indole-5-carbaldehyde (5b). Yellow liquid (2.23 g), 65.0\% yield. ${ }^{1} \mathrm{H}$ NMR $\left(\mathrm{CDCl}_{3}, 500 \mathrm{MHz}\right): \delta 10.00(\mathrm{~s}, 1 \mathrm{H}), 8.12(\mathrm{~d}, J$ $=1.0 \mathrm{~Hz}, 1 \mathrm{H}), 7.77\left(\mathrm{dd},{ }^{3} J=8.5 \mathrm{~Hz},{ }^{4} J=1.0 \mathrm{~Hz}, 1 \mathrm{H}\right), 7.39(\mathrm{~d}, J=$ $8.5 \mathrm{~Hz}, 1 \mathrm{H}), 7.17(\mathrm{~d}, J=3.5 \mathrm{~Hz}, 1 \mathrm{H}), 6.63(\mathrm{~d}, J=3.0 \mathrm{~Hz}, 1 \mathrm{H}), 4.11$ $(\mathrm{t}, J=7.0 \mathrm{~Hz}, 2 \mathrm{H}), 1.83-1.77(\mathrm{~m}, 2 \mathrm{H}), 1.35-1.28(\mathrm{~m}, 2 \mathrm{H}), 0.93(\mathrm{t}, J$ $=7.5 \mathrm{~Hz}, 3 \mathrm{H}) \mathrm{ppm} .{ }^{13} \mathrm{C} \mathrm{NMR}\left(\mathrm{CDCl}_{3}, 125 \mathrm{MHz}\right): \delta 192.2,139.0$, 129.6, 129.1, 128.2, 126.3, 121.4, 109.8, 103.0, 41.2, 32.1, 19.9, 13.4 ppm. Anal. calcd for $\mathrm{C}_{13} \mathrm{H}_{15} \mathrm{NO}: \mathrm{C}, 77.58 ; \mathrm{H}, 7.51 ; \mathrm{N}, 6.96$. Found: C, 77.97; H, 7.48; N, 7.01.

1-Isobutyl-1H-indole-5-carbaldehyde (5c). Yellow liquid $(2.00$ g), 58.3\% yield. ${ }^{1} \mathrm{H}$ NMR $\left(\mathrm{CDCl}_{3}, 500 \mathrm{MHz}\right): \delta 10.02(\mathrm{~s}, 1 \mathrm{H}), 8.14$ $(\mathrm{s}, 1 \mathrm{H}), 7.77(\mathrm{~d}, J=8.5 \mathrm{~Hz}, 1 \mathrm{H}), 7.40(\mathrm{~d}, J=8.5 \mathrm{~Hz}, 1 \mathrm{H}), 7.16(\mathrm{~d}, J$ $=2.5 \mathrm{~Hz}, 1 \mathrm{H}), 6.65(\mathrm{~s}, 1 \mathrm{H}), 3.94(\mathrm{~d}, J=7.5 \mathrm{~Hz}, 2 \mathrm{H}), 2.24-2.15(\mathrm{~m}$, $1 \mathrm{H}), 0.93$ (d, $J=7.0 \mathrm{~Hz}, 6 \mathrm{H}) \mathrm{ppm} .{ }^{13} \mathrm{C} \mathrm{NMR}\left(\mathrm{CDCl}_{3}, 125 \mathrm{MHz}\right)$ : $\delta$ 192.4, 139.6, 130.3, 129.2, 128.2, 126.4, 121.6, 110.1, 103.1, 54.3, 29.7, 20.2 ppm. Anal. calcd for $\mathrm{C}_{13} \mathrm{H}_{15} \mathrm{NO}$ : C, 77.58; H, 7.51; N, 6.96. Found: C, 77.89; H, 7.55; N, 6.99.

1-Dodecyl-1H-indole-5-carbaldehyde (5d). White solid (3.94 g), 73.8\% yield. ${ }^{1} \mathrm{H}$ NMR $\left(\mathrm{CDCl}_{3}, 500 \mathrm{MHz}\right): \delta 10.03(\mathrm{~s}, 1 \mathrm{H}), 8.15$ $(\mathrm{s}, 1 \mathrm{H}), 7.78\left(\mathrm{dd},{ }^{3} J=8.5 \mathrm{~Hz},{ }^{4} J=1.0 \mathrm{~Hz}, 1 \mathrm{H}\right), 7.41(\mathrm{~d}, J=9.0 \mathrm{~Hz}$, $1 \mathrm{H}), 7.19$ (d, $J=3.5 \mathrm{~Hz}, 1 \mathrm{H}), 6.65$ (d, $J=3.5 \mathrm{~Hz}, 1 \mathrm{H}), 4.14(\mathrm{t}, J=$ $7.0 \mathrm{~Hz}, 2 \mathrm{H}), 1.86-1.83(\mathrm{~m}, 2 \mathrm{H}), 1.31-1.24(\mathrm{~m}, 18 \mathrm{H}), 0.88(\mathrm{t}, J=$ $7.0 \mathrm{~Hz}, 3 \mathrm{H}) \mathrm{ppm} .{ }^{13} \mathrm{C} \mathrm{NMR}\left(\mathrm{CDCl}_{3}, 125 \mathrm{MHz}\right): \delta 192.3,139.3$, $129.7,129.3$, 128.3, 126.5, 121.6, 109.9, 103.2, 46.7, 31.9, 30.2, 29.56, 29.49, 29.42, 29.28, 29.15, 26.9, 22.6, 14.1 ppm. Anal. calcd for $\mathrm{C}_{21} \mathrm{H}_{31} \mathrm{NO}$ : C, 80.46; H, 9.97; N, 4.47. Found: C, 79.75; $\mathrm{H}, 10.01 ; \mathrm{N}, 4.50$.

\section{Synthesis of IPM derivatives}

General procedure: Compound $2(172.2 \mathrm{mg}, 1.0 \mathrm{mmol})$ and compound $\mathbf{3} / \mathbf{4} / \mathbf{5}(3.0 \mathrm{mmol})$ were dissolved in the solution of acetonitrile $(10 \mathrm{~mL})$ and piperidine $(0.2 \mathrm{~mL})$. The mixture was heated at $80{ }^{\circ} \mathrm{C}$ under a nitrogen atmosphere for $24 \mathrm{~h}$. After cooling, the mixture was poured into methanol and suction filtered to give the crude product. The crude product was washed with acetone and methanol three times, respectively, and then dried to give the pure target compound. The characterization data for various IPM derivatives are listed as follows:

2-(2,6-Bis((E)-2-(1H-indol-3-yl)vinyl)-4H-pyran-4-ylidene)malononitrile (I3PM). Red solids (362.4 mg), 85.0\% yield. ${ }^{1} \mathrm{H}$ NMR (DMSO- $\left.d_{6}, 500 \mathrm{MHz}\right): \delta 11.87(\mathrm{~s}, 2 \mathrm{H}), 8.25(\mathrm{~d}, J=8.0 \mathrm{~Hz}$, $2 \mathrm{H}), 8.04-7.98(\mathrm{~m}, 4 \mathrm{H}), 7.51(\mathrm{~d}, J=8.0 \mathrm{~Hz}, 2 \mathrm{H}), 7.28-7.19(\mathrm{~m}$, $6 \mathrm{H}), 6.92(\mathrm{~s}, 2 \mathrm{H}) \mathrm{ppm} .{ }^{13} \mathrm{C}$ NMR (DMSO- $\left.d_{6}, 125 \mathrm{MHz}\right): \delta 160.3$, 156.3, 137.6, 132.6, 132.5, 124.6, 122.7, 120.9, 120.8, 116.6, 113.3, 112.7, 112.4, 104.3, 52.6 ppm. MS (ESI, $m / z): 427.10\left(\mathrm{M}^{+}+\right.$ H). Anal. calcd for $\mathrm{C}_{28} \mathrm{H}_{18} \mathrm{~N}_{4} \mathrm{O}: \mathrm{C}, 78.86 ; \mathrm{H}, 4.25 ; \mathrm{N}, 13.14$. Found: C, 79.29; H, 4.22; N, 13.22.

2-(2,6-Bis((E)-2-(1-butyl-1H-indol-3-yl)vinyl)-4H-pyran-4ylidene)malononitrile (I3PMBn). Red solids (483.2 mg), 89.7\% yield. ${ }^{1} \mathrm{H} \mathrm{NMR}\left(\mathrm{CDCl}_{3}, 500 \mathrm{MHz}\right): \delta 7.94(\mathrm{~d}, J=7.5 \mathrm{~Hz}, 2 \mathrm{H}), 7.72$ $(\mathrm{d}, J=15.5 \mathrm{~Hz}, 2 \mathrm{H}), 7.51(\mathrm{~s}, 2 \mathrm{H}), 7.23$ (d, $J=8.0 \mathrm{~Hz}, 2 \mathrm{H}), 7.36-$ $7.30(\mathrm{~m}, 4 \mathrm{H}), 6.67(\mathrm{~d}, J=16.0 \mathrm{~Hz}, 2 \mathrm{H}), 6.48(\mathrm{~s}, 2 \mathrm{H}), 4.19(\mathrm{t}, J=$ $7.0 \mathrm{~Hz}, 4 \mathrm{H}), 1.91-1.87(\mathrm{~m}, 4 \mathrm{H}), 1.43-1.37(\mathrm{~m}, 4 \mathrm{H}), 0.99(\mathrm{t}, J=$ $7.0 \mathrm{~Hz}, 6 \mathrm{H}) \mathrm{ppm} .{ }^{13} \mathrm{C} \mathrm{NMR}\left(\mathrm{CDCl}_{3}, 125 \mathrm{MHz}\right): \delta 159.7,156.2$, 137.6 , 132.1, 131.2, 125.9, 123.2, 121.6, 120.5, 116.5, 113.5, 112.9, 110.5, 104.8, 55.6, 46.7, 32.0, 20.2, 13.6 ppm. MS (ESI, $m$ / z): $539.25\left(\mathrm{M}^{+}+\mathrm{H}\right)$. Anal. calcd for $\mathrm{C}_{36} \mathrm{H}_{34} \mathrm{~N}_{4} \mathrm{O}$ : C, 80.27; $\mathrm{H}, 6.36$; N, 10.40. Found: C, 80.81; H, 6.41; N, 10.49 .

2-(2,6-Bis $((E)-2-(1-i s o b u t y l-1 H$-indol-3-yl)vinyl)-4H-pyran-4ylidene)malononitrile (I3PMBi). Orange-red solids (477.2 mg), $88.6 \%$ yield. ${ }^{1} \mathrm{H}$ NMR $\left(\mathrm{CDCl}_{3}, 500 \mathrm{MHz}\right): \delta$ 7.96-7.94 (m, $2 \mathrm{H}), 7.73\left(\mathrm{dd},{ }^{3} J=16.0 \mathrm{~Hz},{ }^{4} J=3.5 \mathrm{~Hz}, 2 \mathrm{H}\right), 7.49(\mathrm{~d}, J=3.5 \mathrm{~Hz}$, $2 \mathrm{H}), 7.42\left(\mathrm{dd},{ }^{3} J=7.5 \mathrm{~Hz},{ }^{4} J=3.0 \mathrm{~Hz}, 2 \mathrm{H}\right), 7.36-7.27(\mathrm{~m}, 4 \mathrm{H})$, $6.69\left(\mathrm{dd},{ }^{3} J=16.0 \mathrm{~Hz},{ }^{4} J=3.5 \mathrm{~Hz}, 2 \mathrm{H}\right), 6.49(\mathrm{~d}, J=3.5 \mathrm{~Hz}, 2 \mathrm{H})$, $3.39\left(\mathrm{dd},{ }^{3} J=7.0 \mathrm{~Hz},{ }^{4} J=3.5 \mathrm{~Hz}, 4 \mathrm{H}\right), 2.29-2.24(\mathrm{~m}, 2 \mathrm{H}), 0.99$ $\left(\mathrm{dd},{ }^{3} J=6.5 \mathrm{~Hz},{ }^{4} J=3.5 \mathrm{~Hz}, 12 \mathrm{H}\right) \mathrm{ppm} .{ }^{13} \mathrm{C} \mathrm{NMR}\left(\mathrm{CDCl}_{3}, 125\right.$ $\mathrm{MHz}): \delta 159.7,156.2,137.8,132.8,131.3,125.8,123.2,121.6$, $120.5,116.5,113.6,112.7,110.7,104.8,55.5,54.5,29.3$, $20.3 \mathrm{ppm}$. MS (ESI, $\mathrm{m} / \mathrm{z}): 539.25\left(\mathrm{M}^{+}+\mathrm{H}\right)$. Anal. calcd for $\mathrm{C}_{36} \mathrm{H}_{34} \mathrm{~N}_{4} \mathrm{O}$ : C, 80.27; H, 6.36; N, 10.40. Found: C, 80.92; H, 6.39; $\mathrm{N}, 10.50$.

2-(2,6-Bis((E)-2-(1-dodecyl-1H-indol-3-yl)vinyl)-4H-pyran-4ylidene)malononitrile (I3PMD). Orange-red solids (662.3 $\mathrm{mg}$ ), 86.8\% yield. ${ }^{1} \mathrm{H}$ NMR $\left(\mathrm{CDCl}_{3}, 500 \mathrm{MHz}\right): \delta 7.94(\mathrm{~d}, J=8.0 \mathrm{~Hz}$, $2 \mathrm{H}), 7.72(\mathrm{~d}, J=15.5 \mathrm{~Hz}, 2 \mathrm{H}), 7.50(\mathrm{~s}, 2 \mathrm{H}), 7.42(\mathrm{~d}, J=8.5 \mathrm{~Hz}$, 2H), 7.36-7.29 (m, 4H), $6.67(\mathrm{~d}, J=16.0 \mathrm{~Hz}, 2 \mathrm{H}), 6.47(\mathrm{~s}, 2 \mathrm{H})$, $4.17(\mathrm{t}, J=7.0 \mathrm{~Hz}, 4 \mathrm{H}), 1.92-1.89(\mathrm{~m}, 4 \mathrm{H}), 1.35-1.25(\mathrm{~m}, 36 \mathrm{H})$, $0.87(\mathrm{t}, J=7.0 \mathrm{~Hz}, 6 \mathrm{H}) \mathrm{ppm} .{ }^{13} \mathrm{C} \mathrm{NMR}\left(\mathrm{CDCl}_{3}, 125 \mathrm{MHz}\right): \delta 159.7$, 156.2 , 137.6, 132.1, 131.2, 125.9, 123.2, 121.6, 120.5, 116.4, 113.5, 112.9, 110.5, 104.7, 55.6, 47.0, 31.9, 30.0, 29.6, 29.5, 29.4, 29.3, 29.2, 27.0, 22.7, $14.1 \mathrm{ppm}$. MS (ESI, $m / z)$ : $763.45\left(\mathrm{M}^{+}+\mathrm{H}\right)$. Anal. calcd for $\mathrm{C}_{52} \mathrm{H}_{66} \mathrm{~N}_{4} \mathrm{O}$ : C, 81.84; H, 8.72; N, 7.34. Found: C, $82.34 ; \mathrm{H}, 8.67 ; \mathrm{N}, 7.40$.

2-(2,6-Bis((E)-2-(1H-indol-4-yl)vinyl)-4H-pyran-4-ylidene)malononitrile (I4PM). Orange-red solids (300.1 mg), 70.4\% yield. ${ }^{1} \mathrm{H}$ NMR (DMSO- $\left.d_{6}, 500 \mathrm{MHz}\right): \delta 11.50(\mathrm{~s}, 2 \mathrm{H}), 8.06(\mathrm{~d}, J=$ $16.5 \mathrm{~Hz}, 2 \mathrm{H}), 7.58-7.46(\mathrm{~m}, 8 \mathrm{H}), 7.21$ (d, $J=7.5 \mathrm{~Hz}, 2 \mathrm{H}), 7.10$ (s, $4 \mathrm{H})$ ppm. ${ }^{13} \mathrm{C}$ NMR (DMSO- $\left.d_{6}, 125 \mathrm{MHz}\right): \delta 159.3,156.3,137.4$, 136.5, 127.1, 126.4, 126.0, 121.6, 121.1, 118.6, 115.9, 114.0, 106.9, 100.6, 55.7 ppm. MS (ESI, $m / z): 427.10\left(\mathrm{M}^{+}+\mathrm{H}\right)$. Anal. calcd for $\mathrm{C}_{28} \mathrm{H}_{18} \mathrm{~N}_{4} \mathrm{O}: \mathrm{C}, 78.86 ; \mathrm{H}, 4.25 ; \mathrm{N}, 13.14$. Found: $\mathrm{C}$, $79.25 ; \mathrm{H}, 4.23 ; \mathrm{N}, 13.02$. 
2-(2,6-Bis((E)-2-(1-butyl-1H-indol-4-yl)vinyl)-4H-pyran-4ylidene)malononitrile (I4PMBn). Orange-red solids (430.4 mg), 79.9\% yield. ${ }^{1} \mathrm{H} \mathrm{NMR}\left(\mathrm{CDCl}_{3}, 500 \mathrm{MHz}\right): \delta 7.92(\mathrm{~d}, J=16.0 \mathrm{~Hz}$, $2 \mathrm{H}), 7.42(\mathrm{~d}, J=8.5 \mathrm{~Hz}, 2 \mathrm{H}), 7.37$ (d, $J=7.0 \mathrm{~Hz}, 2 \mathrm{H}), 7.28-7.23$ $(\mathrm{m}, 4 \mathrm{H}), 6.86(\mathrm{~d}, J=16.0 \mathrm{~Hz}, 2 \mathrm{H}), 6.77(\mathrm{~d}, J=2.5 \mathrm{~Hz}, 2 \mathrm{H}), 6.55(\mathrm{~s}$, $2 \mathrm{H}), 4.16(\mathrm{t}, J=7.0 \mathrm{~Hz}, 4 \mathrm{H}), 1.87-1.81(\mathrm{~m}, 4 \mathrm{H}), 1.40-1.32(\mathrm{~m}$, $4 \mathrm{H}), 0.95$ (t, $J=7.0 \mathrm{~Hz}, 6 \mathrm{H}) \mathrm{ppm} .{ }^{13} \mathrm{C} \mathrm{NMR}\left(\mathrm{CDCl}_{3}, 125 \mathrm{MHz}\right)$ : $\delta$ 158.6, 156.0, 136.9, 136.6, 129.6, 127.5, 126.8, 121.6, 119.9, 118.3, 115.6, 111.9, 106.7, 99.3, 58.6, 46.4, 32.4, 20.2, 13.6 ppm. MS (EI, m/z): $538.29\left(\mathrm{M}^{+}, 56 \%\right), 495.23$ (8\%), 394.25 (22\%), 226.10 (57\%), 186.12 (49\%). Anal. calcd for $\mathrm{C}_{36} \mathrm{H}_{34} \mathrm{~N}_{4} \mathrm{O}: \mathrm{C}, 80.27$; H, 6.36; N, 10.40. Found: C, 80.83; H, 6.33; N, 10.32.

2-(2,6-Bis $((E)-2-(1-i s o b u t y l-1 H$-indol-4-yl)vinyl)-4H-pyran-4ylidene)malononitrile (I4PMBi). Orange-red solids $(360.7 \mathrm{mg})$, $67.0 \%$ yield. ${ }^{1} \mathrm{H} \mathrm{NMR}\left(\mathrm{CDCl}_{3}, 500 \mathrm{MHz}\right): \delta 7.99(\mathrm{~d}, J=16.0 \mathrm{~Hz}$, $2 \mathrm{H}), 7.42\left(\mathrm{dd},{ }^{3} J=14.0 \mathrm{~Hz},{ }^{4} J=8.0 \mathrm{~Hz}, 4 \mathrm{H}\right), 7.29-7.27(\mathrm{~m}, 4 \mathrm{H})$, $6.93(\mathrm{~d}, J=16.0 \mathrm{~Hz}, 2 \mathrm{H}), 6.82(\mathrm{~d}, J=2.5 \mathrm{~Hz}, 2 \mathrm{H}), 6.64(\mathrm{~s}, 2 \mathrm{H})$, $3.99(\mathrm{~d}, J=7.5 \mathrm{~Hz}, 4 \mathrm{H}), 2.28-2.20(\mathrm{~m}, 2 \mathrm{H}), 0.97(\mathrm{~d}, J=6.5 \mathrm{~Hz}$, $12 \mathrm{H}) \mathrm{ppm} .{ }^{13} \mathrm{C} \mathrm{NMR}\left(\mathrm{CDCl}_{3}, 125 \mathrm{MHz}\right): \delta$ 158.7, 156.0, 137.0, $136.9,130.2$, 127.4, 126.9, 121.6, 120.0, 118.4, 115.6, 112.1, 106.7, 99.2, 58.8, 54.4, 29.7, 20.3 ppm. MS (ESI, $m / z): 539.25\left(\mathrm{M}^{+}\right.$ $+\mathrm{H}$ ). Anal. calcd for $\mathrm{C}_{36} \mathrm{H}_{34} \mathrm{~N}_{4} \mathrm{O}$ : C, 80.27; H, 6.36; N, 10.40 . Found: C, 79.86; H, 6.32; N, 10.35 .

2-(2,6-Bis $((E)-2-(1-d o d e c y l-1 H$-indol-4-yl)vinyl)-4H-pyran-4ylidene)malononitrile (I4PMD). Orange-red solids $(600.3 \mathrm{mg})$, 78.7\% yield. ${ }^{1} \mathrm{H} \mathrm{NMR}\left(\mathrm{CDCl}_{3}, 500 \mathrm{MHz}\right): \delta 7.94(\mathrm{~d}, J=16.0 \mathrm{~Hz}$, $2 \mathrm{H}), 7.39\left(\mathrm{dd},{ }^{3} J=19.0 \mathrm{~Hz},{ }^{4} J=7.0 \mathrm{~Hz}, 4 \mathrm{H}\right), 7.26(\mathrm{~d}, J=3.5 \mathrm{~Hz}$, $2 \mathrm{H}), 7.24(\mathrm{~d}, J=7.0 \mathrm{~Hz}, 2 \mathrm{H}), 6.88(\mathrm{~d}, J=16.0 \mathrm{~Hz}, 2 \mathrm{H}), 6.77(\mathrm{~d}, J=$ $2.5 \mathrm{~Hz}, 2 \mathrm{H}), 6.59(\mathrm{~s}, 2 \mathrm{H}), 4.14(\mathrm{t}, J=7.0 \mathrm{~Hz}, 4 \mathrm{H}), 1.86-1.83(\mathrm{~m}$, $4 \mathrm{H}), 1.31-1.23(\mathrm{~m}, 36 \mathrm{H}), 0.85(\mathrm{t}, J=6.5 \mathrm{~Hz}, 4 \mathrm{H}) \mathrm{ppm} .{ }^{13} \mathrm{C} \mathrm{NMR}$ $\left(\mathrm{CDCl}_{3}, 125 \mathrm{MHz}\right): \delta$ 158.7, 156.0, 137.0, 136.6, 129.6, 127.5, 126.9, 121.6, 120.0, 118.4, 115.6, 111.9, 106.7, 99.3, 58.7, 46.7, 31.9, 30.3, 29.6, 29.5, 29.4, 29.3, 29.2, 27.0, 22.7, 14.1 ppm. MS (ESI, $m / z)$ : $763.45\left(\mathrm{M}^{+}+\mathrm{H}\right)$. Anal. calcd for $\mathrm{C}_{52} \mathrm{H}_{66} \mathrm{~N}_{4} \mathrm{O}$ : C, 81.84; H, 8.72; N, 7.34. Found: C, 82.16; H, 8.77; N, 7.38.

2-(2,6-Bis((E)-2-(1H-indol-5-yl)vinyl)-4H-pyran-4-ylidene) malononitrile (I5PM). Orange-red solids (311.7 mg), 73.1\% yield. ${ }^{1} \mathrm{H}$ NMR (DMSO- $\left.d_{6}, 500 \mathrm{MHz}\right): \delta 11.39(\mathrm{~s}, 2 \mathrm{H}), 8.07(\mathrm{~s}, 2 \mathrm{H})$, $7.92(\mathrm{~d}, J=16.0 \mathrm{~Hz}, 2 \mathrm{H}), 7.63(\mathrm{~d}, J=16.5 \mathrm{~Hz}, 2 \mathrm{H}), 7.48(\mathrm{~d}, J=$ $8.5 \mathrm{~Hz}, 2 \mathrm{H}), 7.43$ (s, 2H), 7.29 (d, $J=16.0 \mathrm{~Hz}, 2 \mathrm{H}), 6.81(\mathrm{~s}, 2 \mathrm{H})$, $6.54(\mathrm{~s}, 2 \mathrm{H}) \mathrm{ppm} .{ }^{13} \mathrm{C}$ NMR (DMSO- $\left.d_{6}, 125 \mathrm{MHz}\right): \delta 159.6,156.2$, $140.0,137.1,128.0,126.7,126.3$, 122.5, 120.6, 116.0, 115.5, 112.1, 105.7, 102.1, 54.7 ppm. MS (ESI, $m / z): 427.10\left(\mathrm{M}^{+}+\mathrm{H}\right)$. Anal. calcd for $\mathrm{C}_{28} \mathrm{H}_{18} \mathrm{~N}_{4} \mathrm{O}: \mathrm{C}, 78.86 ; \mathrm{H}, 4.25 ; \mathrm{N}, 13.14$. Found: $\mathrm{C}$, $78.51 ; \mathrm{H}, 4.28 ; \mathrm{N}, 13.06$.

2-(2,6-Bis((E)-2-(1-butyl-1H-indol-5-yl)vinyl)-4H-pyran-4ylidene)malononitrile (I5PMBn). Orange solids (245.2 $\mathrm{mg}$ ), $45.5 \%$ yield. ${ }^{1} \mathrm{H} \mathrm{NMR}\left(\mathrm{CDCl}_{3}, 500 \mathrm{MHz}\right): \delta 7.84(\mathrm{~s}, 2 \mathrm{H}), 7.67(\mathrm{~d}, J$ $=16.0 \mathrm{~Hz}, 2 \mathrm{H}), 7.48(\mathrm{~d}, J=8.5 \mathrm{~Hz}, 2 \mathrm{H}), 7.38(\mathrm{~d}, J=8.5 \mathrm{~Hz}, 2 \mathrm{H})$, $7.15(\mathrm{~s}, 2 \mathrm{H}), 6.69(\mathrm{~d}, J=16.0 \mathrm{~Hz}, 2 \mathrm{H}), 6.58-6.56(\mathrm{~m}, 4 \mathrm{H}), 4.14(\mathrm{t}, J$ $=7.5 \mathrm{~Hz}, 4 \mathrm{H}), 1.88-1.82(\mathrm{~m}, 4 \mathrm{H}), 1.40-1.34(\mathrm{~m}, 4 \mathrm{H}), 0.97(\mathrm{t}, J=$ $7.5 \mathrm{~Hz}, 6 \mathrm{H}) \mathrm{ppm} .{ }^{13} \mathrm{C} \mathrm{NMR}\left(\mathrm{CDCl}_{3}, 125 \mathrm{MHz}\right): \delta$ 159.1, 156.2, $139.8,137.3,129.2$, 129.0, 126.2, 122.6, 120.6, 115.8, 115.3, 110.2, 106.0, 102.2, 57.6, 46.4, 32.4, 20.1, 13.6 ppm. MS (ESI, $m$ / z): $539.25\left(\mathrm{M}^{+}+\mathrm{H}\right)$. Anal. calcd for $\mathrm{C}_{36} \mathrm{H}_{34} \mathrm{~N}_{4} \mathrm{O}$ : C, 80.27; $\mathrm{H}, 6.36$; N, 10.40. Found: C, 79.75; H, 6.31; N, 10.33.
2-(2,6-Bis((E)-2-(1-isobutyl-1H-indol-5-yl)vinyl)-4H-pyran-4ylidene)malononitrile (I5PMBi). Brown solids (223.7 mg), 41.5\% yield. ${ }^{1} \mathrm{H} \mathrm{NMR}\left(\mathrm{CDCl}_{3}, 500 \mathrm{MHz}\right): \delta 7.85(\mathrm{~s}, 2 \mathrm{H}), 7.68(\mathrm{~d}, J=$ $15.5 \mathrm{~Hz}, 2 \mathrm{H}), 7.48(\mathrm{~d}, J=8.5 \mathrm{~Hz}, 2 \mathrm{H}), 7.38(\mathrm{~d}, J=8.5 \mathrm{~Hz}, 2 \mathrm{H})$, $7.13(\mathrm{~s}, 2 \mathrm{H}), 6.70$ (d, $J=15.5 \mathrm{~Hz}, 2 \mathrm{H}), 6.59(\mathrm{~d}, J=16.0 \mathrm{~Hz}, 4 \mathrm{H})$, $3.94(\mathrm{~d}, J=7.5 \mathrm{~Hz}, 4 \mathrm{H}), 2.25-2.19(\mathrm{~m}, 2 \mathrm{H}), 0.96$ (d, $J=6.5 \mathrm{~Hz}$, $12 \mathrm{H}) \mathrm{ppm} .{ }^{13} \mathrm{C} \mathrm{NMR}\left(\mathrm{CDCl}_{3}, 125 \mathrm{MHz}\right): \delta$ 159.1, 156.2, 139.8, $137.6,129.8,129.0,126.3,122.6$, 120.6, 115.8, 115.4, 110.5, 106.0, 102.1, 57.7, 54.3, 29.7, 20.3 ppm. MS (ESI, $\mathrm{m} / \mathrm{z}$ ): 539.25 $\left(\mathrm{M}^{+}+\mathrm{H}\right)$. Anal. calcd for $\mathrm{C}_{36} \mathrm{H}_{34} \mathrm{~N}_{4} \mathrm{O}$ : C, 80.27; $\mathrm{H}, 6.36 ; \mathrm{N}, 10.40$. Found: C, 79.69; H, 6.33; N, 10.48 .

2-(2,6-Bis((E)-2-(1-dodecyl-1H-indol-5-yl)vinyl)-4H-pyran-4ylidene)malononitrile (I5PMD). Brown solids (162.7 mg), 21.3\% yield. ${ }^{1} \mathrm{H} \mathrm{NMR}\left(\mathrm{CDCl}_{3}, 500 \mathrm{MHz}\right): \delta 7.85(\mathrm{~s}, 2 \mathrm{H}), 7.70(\mathrm{~d}, J=$ $15.5 \mathrm{~Hz}, 2 \mathrm{H}), 7.49$ (d, $J=8.5 \mathrm{~Hz}, 2 \mathrm{H}), 7.39$ (d, $J=8.5 \mathrm{~Hz}, 2 \mathrm{H})$, 7.15 (s, 2H), 6.72 (d, $J=16.0 \mathrm{~Hz}, 2 \mathrm{H}), 6.63(\mathrm{~s}, 2 \mathrm{H}), 6.57(\mathrm{~s}, 2 \mathrm{H})$, $4.14(\mathrm{t}, J=7.0 \mathrm{~Hz}, 4 \mathrm{H}), 1.88-1.85(\mathrm{~m}, 4 \mathrm{H}), 1.33-1.26(\mathrm{~m}, 36 \mathrm{H})$, $0.86(\mathrm{t}, J=6.5 \mathrm{~Hz}, 6 \mathrm{H}) \mathrm{ppm} .{ }^{13} \mathrm{C} \mathrm{NMR}\left(\mathrm{CDCl}_{3}, 125 \mathrm{MHz}\right): \delta 159.1$, 156.2 , 139.9, 137.3, 129.2, 129.1, 126.3, 122.6, 120.7, 115.8, 115.4, 110.3, 106.0, 102.2, 57.8, 46.7, 31.9, 30.3, 29.6, 29.5, 29.4, 29.3, 29.2, 27.0, 22.7, 14.1 ppm. MS (EI, $m / z): 761.24\left(\mathrm{M}^{+}-\mathrm{H}\right.$, 100), 382.23 (31\%), 298.23 (78\%), 207.03 (69\%), 186.10 (73\%), 158.08 (67\%). Anal. calcd for $\mathrm{C}_{52} \mathrm{H}_{66} \mathrm{~N}_{4} \mathrm{O}: \mathrm{C}, 81.84 ; \mathrm{H}, 8.72 ; \mathrm{N}$, 7.34. Found: C, 81.23; H, 8.69; N, 7.29.

\section{Results and discussion}

\section{Synthesis and features of IPM derivatives}

The chemical structures and synthetic route of IPM derivatives are depicted in Scheme 1. The intermediate 2-(2,6-dimethyl-4Hpyran-4-ylidene)malononitrile (2) was synthesized according to the previous literature procedure using 2,6-dimethyl-4-pyrone (1) as the starting material. ${ }^{20}$ The intermediates $\mathbf{3 b} \mathbf{b} \mathbf{3 d} / \mathbf{4} \mathbf{b}-\mathbf{4 d}$ / $\mathbf{5 b}-\mathbf{5 d}$ were obtained from the alkylation of $\mathbf{3 a} / \mathbf{4 a} / \mathbf{5 a}$ with the corresponding alkyl bromide in the presence of $\mathrm{KOH}$, respectively, adopting a method similar to the previous literature procedures (Scheme S1, ESI $\dagger$ ). ${ }^{21}$ And then the IPM derivatives could be facilely prepared from a Knoevenagel reaction of the intermediate $\mathbf{2}$ with $\mathbf{3 a}-\mathbf{3} \mathbf{d} / \mathbf{4 a}-\mathbf{4} \mathbf{d} / \mathbf{5 a}-\mathbf{5 d}$, respectively. The chemical structures of IPM derivatives were confirmed by NMR spectroscopy, mass spectrometry, and elemental analysis. Among these compounds, the structures of I3PM, I3PMBn, I3PMBi, I4PMBn, I4PMBi, and I5PM were further confirmed by single-crystal X-ray diffraction analysis. These target compounds are soluble in common organic solvents, such as chloroform, dichloromethane, toluene, tetrahydrofuran (THF), and ethyl acetate (EA).

\section{Photophysical properties of IPM derivatives in solution and solid state}

The absorption and fluorescence spectra of the IPM derivatives in THF solution were measured as shown in Fig. S1 and S2 (ESI $\dagger$ ), respectively, and the data are presented in Table S1 (ESI $\dagger$ ). The IPM derivatives in THF solution have similar absorption and emission spectra, and exhibit three or four absorption peaks in the range of 333-487 $\mathrm{nm}$, and one emission 


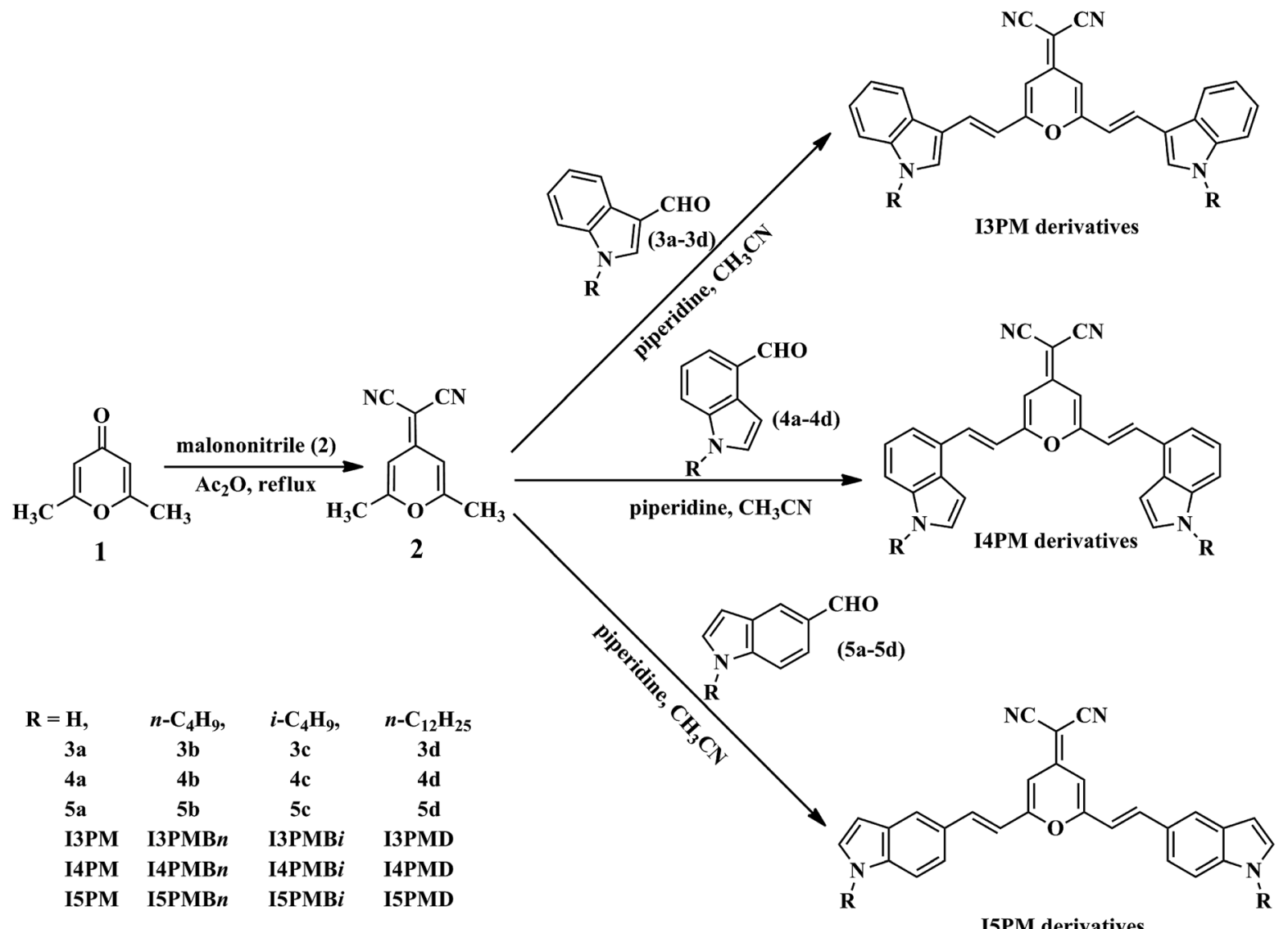

Scheme 1 Chemical structures and synthetic routes of IPM derivatives.

peaks in the range of 529-580 $\mathrm{nm}$. Compared with I3PM, I4PM, and I5PM, the corresponding $N$-alkylated IPM derivatives show a slight redshift in absorption spectra as well as fluorescence spectra because of the introduction of the electron-donating alkyl group. However, the different alkyl groups or isomerized indole units do not show obvious influence on their absorption and fluorescence spectra in THF solution.

Furthermore, the fluorescence spectra of the IPM derivatives in the solid state were investigated. As shown in Fig. S3 (ESI $\dagger$ ) and Table 1, the original solid samples of I3PM, I4PM, and I5PM emit red or orange fluorescence, and appear emission peaks at 634, 607, and $608 \mathrm{~nm}$, respectively. Compared with I3PM and I4PM, the corresponding $N$-alkylated IPM derivatives in the solid state exhibit an obvious blue shift in emission spectra, whereas, the emission spectra I5PM derivatives are related irregularly to the change of the alkyl chain. It is worth mentioning that the increase of alkyl chain length (from $n$-butyl group to $n$-dodecyl group) leads to the blue shift for the I3PM derivatives and the red shift for the I4PM derivatives in the emission spectra, showing an interesting correlation with their MFC behaviors which will be mentioned below. Contrary to the situation in the solution, the alkyl groups exhibit an obvious influence on the solid-state fluorescence properties of IPM derivatives.

\section{MFC properties of IPM derivatives}

The MFC behaviors of the original samples of IPM derivatives were explored (Fig. 1). When the original solid samples of I3PM derivatives were put in a mortar and ground with a pestle, the change of the fluorescence color was not obvious for I3PM and I3PMBn. Although grinding led to an obvious red shift in the emission spectra of I3PM and I3PMBn (Fig. 2 and Table 1) and

Table 1 Emission wavelengths of the IPM derivatives in solid state

\begin{tabular}{lllcl}
\hline Compound & $\lambda_{\text {Original }}(\mathrm{nm})$ & $\lambda_{\text {Ground }}(\mathrm{nm})$ & $\Delta \lambda_{\mathrm{MC}}{ }^{a}(\mathrm{~nm})$ & $\lambda_{\text {Fumed }}(\mathrm{nm})$ \\
\hline I3PM & 634 & 655 & 21 & n.d. $^{b}$ \\
I3PMB $n$ & 622 & 631 & 9 & n.d. \\
I3PMBi & 601 & 632 & 31 & 618 \\
I3PMD & 591 & 604 & 13 & 592 \\
I4PM & 607 & 634 & 27 & 613 \\
I4PMB $n$ & 588 & 621 & 33 & 583 \\
I4PMBi & 586 & 626 & 40 & 585 \\
I4PMD & 590 & 599 & 9 & n.d. \\
I5PM & 608 & 618 & 10 & 615 \\
I5PMBn & 613 & 614 & 1 & n.d. \\
I5PMBi & $588,675(\mathrm{~s})^{c}$ & $593,675(\mathrm{~s})$ & 5 & n.d. \\
I5PMD & $603,657(\mathrm{~s})$ & $603,663(\mathrm{~s})$ & 6 & n.d.
\end{tabular}

${ }^{a} \lambda_{\mathrm{MC}}=\lambda_{\text {Ground }}-\lambda_{\text {Original. }}{ }^{b}$ n.d. $=$ no detection. ${ }^{c} \mathrm{~s}=$ shoulder peak. 


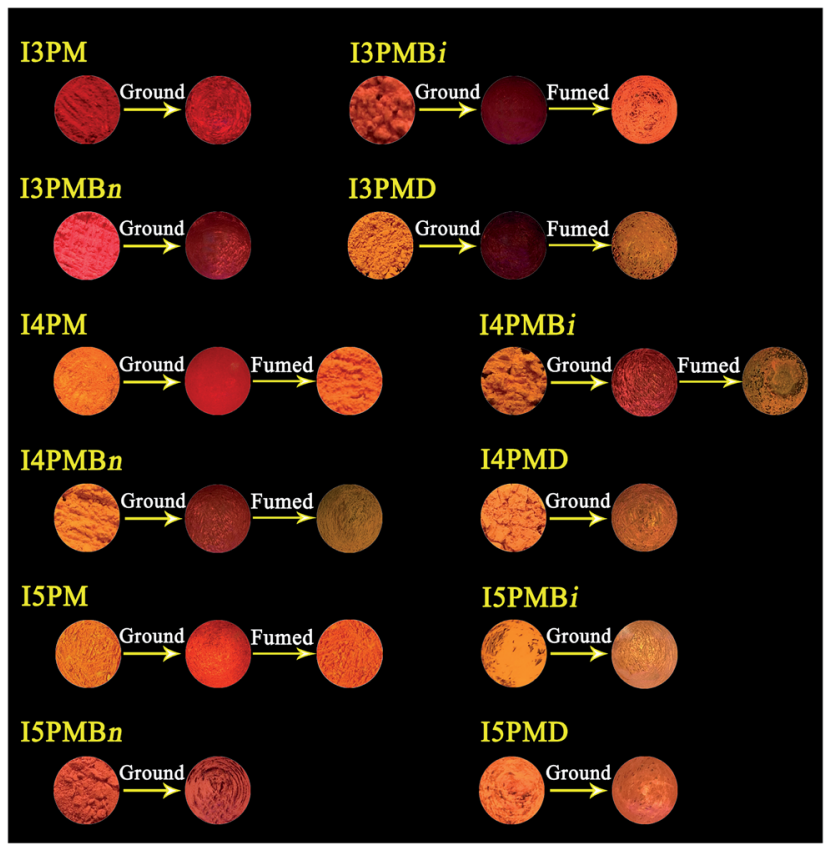

Fig. 1 Photographs of the solid samples of IPM derivatives upon pressing and solvent-fuming (EA vapor) under a $365 \mathrm{~nm}$ UV lamp.

the grinding-induced spectral shifts $\left(\Delta \lambda_{\mathrm{MFC}}=\lambda_{\text {Ground }}-\lambda_{\text {Original }}\right)$ were 21 and $9 \mathrm{~nm}$, respectively, these two compounds did not show obvious MFC phenomena because the fluorescence color change from red to more red could not be well recognized by the naked eye. However, a color change was clearly observed from orange to red for I3PMBi $\left(\Delta \lambda_{\mathrm{MFC}}=31 \mathrm{~nm}\right)$ and I3PMD $\left(\Delta \lambda_{\mathrm{MFC}}=\right.$ $13 \mathrm{~nm}$ ), and the fluorescence colors of their ground samples could be changed into orange by solvent-fuming (exposed to EA vapor), suggesting these two compounds exhibited reversible MFC properties. The results indicated that the isomerization of the alkyl chain and the increase of the alkyl chain length were favorable to the effective mechanofluorochromism of the I3PM derivatives.

For I4PM derivatives, by grinding the original samples of I4PM, I4PMBn, and I4PMBi with orange fluorescence, the corresponding red-emitting ground samples were obtained and their $\Delta \lambda_{\text {MFC }}$ values were 27,33 , and $31 \mathrm{~nm}$, respectively. Different from I3PMD, I4PMD containing a dodecyl chain did not show the fluorescence color change in response to press stimuli, indicating that the increase of the alkyl chain length were disadvantageous to the MFC properties of the I4PM derivatives. As for I5PM derivatives, interestingly, only the original samples of I5PM exhibited reversible fluorescence color change upon grinding $\left(\Delta \lambda_{\mathrm{MFC}}=10 \mathrm{~nm}\right)$ and solvent-fuming, the fluorescence spectra and colors of the other homologues had no significant change $\left(\Delta \lambda_{\mathrm{MFC}}=5-6 \mathrm{~nm}\right)$. Thereby the introduction of the alkyl chain was not advantageous for the MFC properties of the I5PM derivatives. As mentioned above, the alkyl chains exhibited different effects on the MFC properties of the IPM derivatives when the substitution position of the indole unit was different.

To understand the different MC behaviors of the IPM derivatives upon external stimuli, XRD measurements were
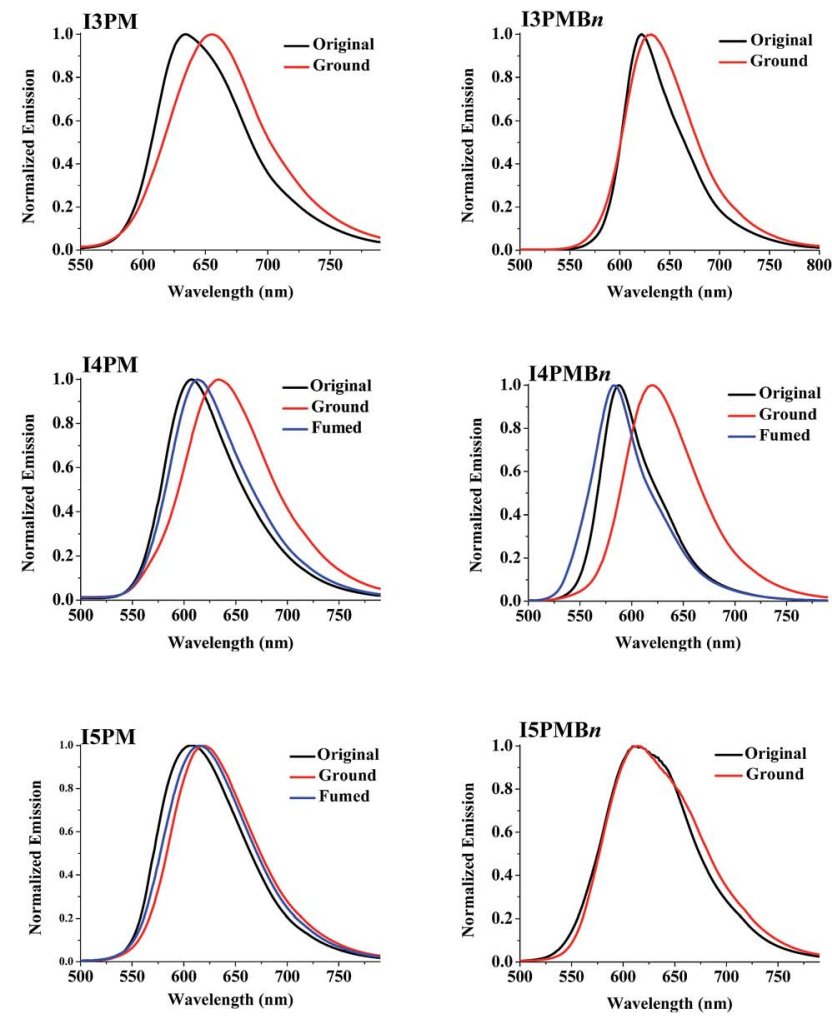
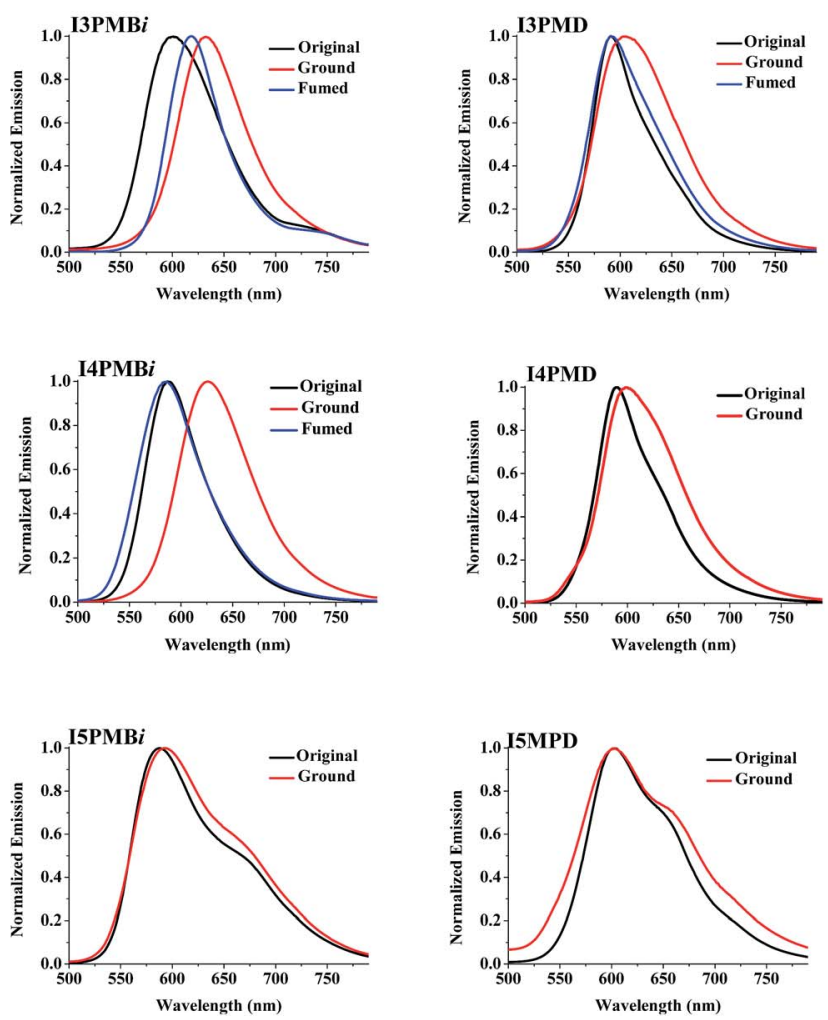

Fig. 2 Normalized fluorescence spectra of the solid samples of IPM derivatives upon external stimuli. 
carried out for the samples (Fig. 3, S4, and S5, ESI $\dagger$ ). As shown in Fig. 3, XRD measurements indicated all the I3PM derivatives showed different structures of molecular aggregation before and after grinding. The XRD curves of the original samples of I3PMBi and I3PMD displayed sharp and intense diffraction peaks, indicating they were crystalline structures. These diffraction peaks disappeared or decreased obviously after grinding, indicating the destruction of crystalline structures and the formation of amorphous structures. Thus, we could conclude that the original samples underwent a morphological change from a crystalline state to an amorphous state upon grinding. The diffraction peaks were recovered when the ground samples were fumed by EA vapor and the recovery led to the blue-shifted fluorescence spectra as well as the recovery of the fluorescence colors. It was noted that the morphology of I3PM and I3PMB was also significantly changed under the stimulation of pressure, which was consistent with the redshifted emission wavelengths observed in the fluorescence spectra (Fig. 2), but the fluorescence color change was hardly distinguished. For the I4PM and I5PM derivatives, the grinding treatment led to the transformation from crystalline state to amorphous state of the MFC-active compounds, such as I4PM, I4PMBn, I4PMBi, and I5PM, whereas, the morphology of the MFC-inactive compounds including I4PMD, I5PMBn, I5PMBi, and I5PMD still maintained (Fig. S4 and S5, ESI $\dagger$ ). The results indicated the MFC properties of the IPM derivatives should be mainly attributed to the transformation between amorphous and crystalline states.

\section{Crystal structures of IPM derivatives}

To gain deep insight into the underlying reasons for the photophysical behavior of the IPM derivatives in the solid state, single crystals of some of the target compounds were obtained and the crystal structure analysis was performed..$^{22}$ Single crystals of I3PM and I5PM were obtained from a THF/1,4-dioxane solution $(\mathrm{v} / \mathrm{v}=1: 1)$, and those of I3PMBn, I3PMBi, I4PMBn, and I4PMBi were cultured from a $\mathrm{CHCl}_{3} /$ ethanol solution $(\mathrm{v} / \mathrm{v}=$ $1: 1$ ), respectively. The crystal structures of these compounds are shown in Fig. 4 and the selected crystal data are given in Table 2. The unit cells of I3PM, I3PMBn, I4PMBi, and I5PM are all triclinic with space group $P \overline{1}$, and those of I3PMBi and I4PMB $n$ are monoclinic with space group $P 12_{1} / c 1$ and $C 2 / c$, respectively.

For I3PM, the dihedral angles between the $4 H$-pyran cycle and the two indole units are $3.61^{\circ}$ and $9.64^{\circ}$, respectively, indicating its molecule shows a certain degree of distortion. And there are no obvious $\pi-\pi$ stacking interactions in the crystal of I3PM. One kind of aliphatic $\mathrm{C}-\mathrm{H} \cdots \mathrm{N}$ bonds $(2.688 \AA)$ restrict the two adjacent I3PM molecules in the same layer (Fig. 5 and Table 3). Because of the unit cell of I3PM includes three 1,4-dioxane molecules, these solvent molecules connect the I3PM molecules of the upper and lower layers by two kinds of $\mathrm{C}-\mathrm{H} \cdots \mathrm{O}$ bonds $(2.645$ and $2.687 \AA$ ), one kind of $\mathrm{N}-\mathrm{H} \cdots \mathrm{O}$ hydrogen bond (2.899 $\AA$ ), and $\mathrm{C}-\mathrm{H} \cdots \pi$ bond $(2.796 \AA)$. The intermolecular interactions in crystal I3PM promote the formation of a stairs-appearance structure. In comparison with I3PM, I5PM has a more twisted conformation because the
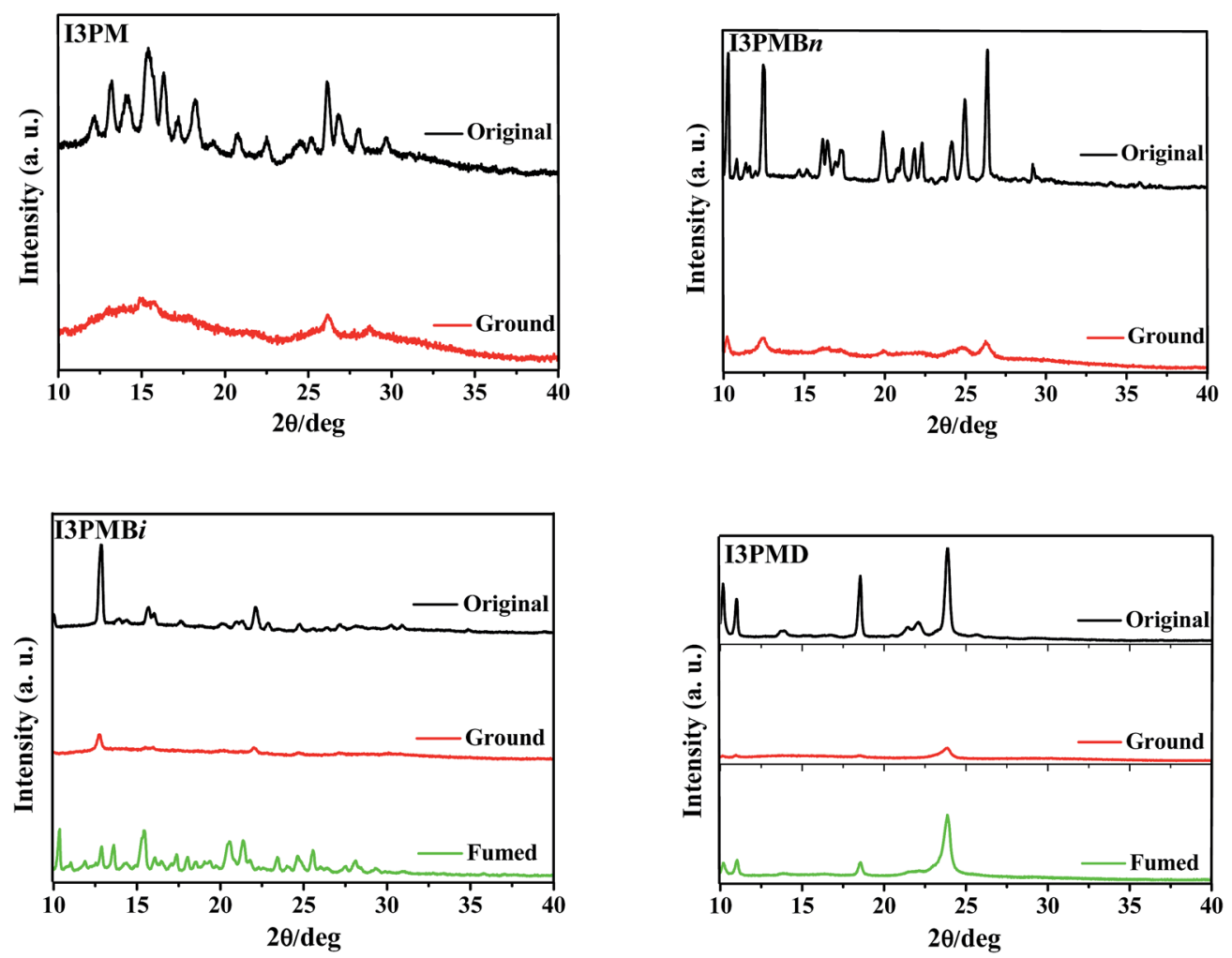

Fig. 3 XRD curves of the solid samples of I3PM derivatives upon external stimuli. 

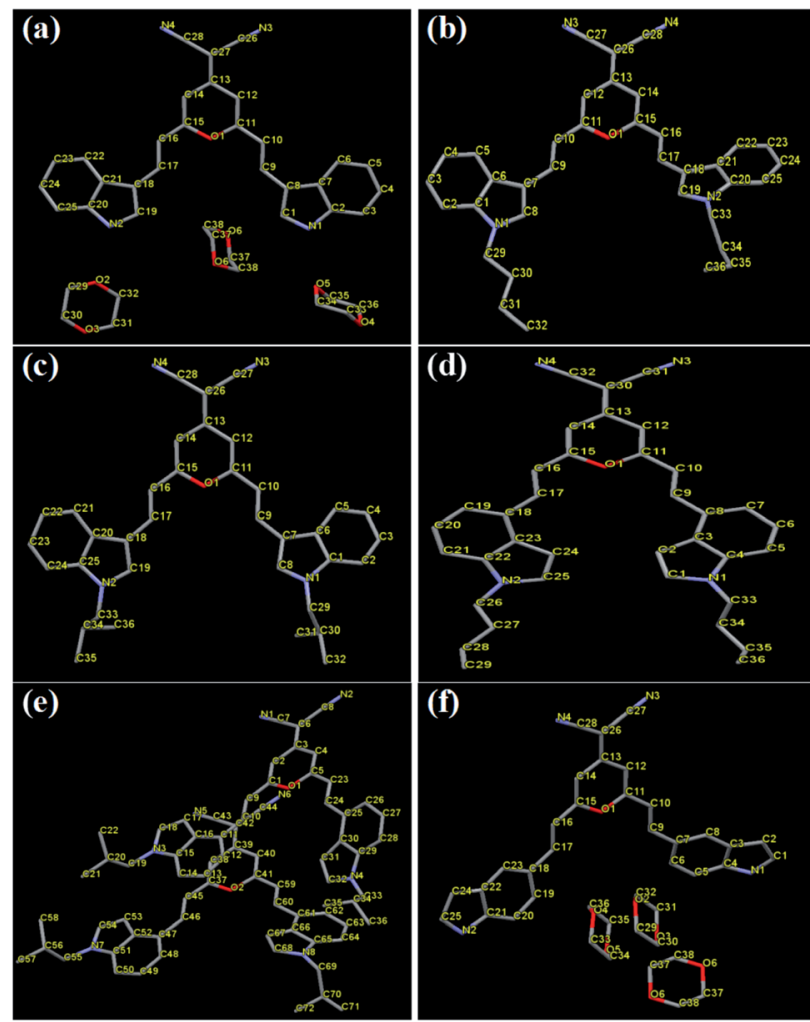

Fig. 4 Single-crystal structures of I3PM (a), I3PMBn (b), I3PMBi (c), I4PMBn (d), I4PMBi (e), and I5PM (f). Hydrogen atoms are omitted for clarity.

dihedral angle between the $4 H$-pyran cycle and one of the indole units reaches $27.82^{\circ}$, which can use as an explanation for the facts that I5PM has blue-shifted absorption and emission
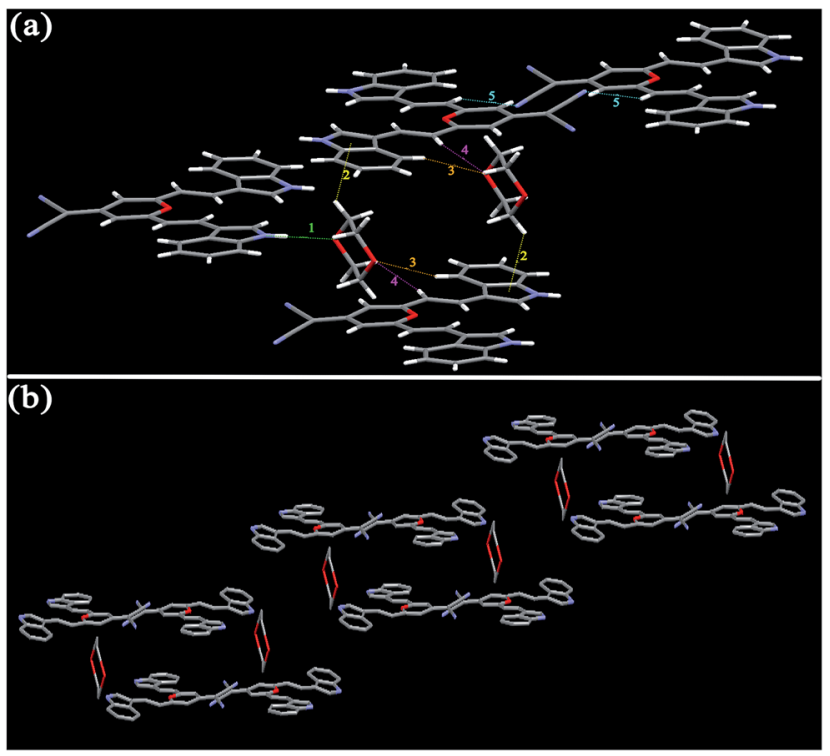

Fig. 5 Intermolecular interactions (a) and molecular packing (b) in I3PM.

spectra in THF solution (Table $\mathrm{S} 1 \dagger$ ) as well as in solid state (Table 1 ). There are weak $\pi-\pi$ stacking in crystal I5PM because the distances between the indole ring and the $4 H$-pyran ring in the upper and lower layers are measured to be 3.642 and $3.698 \AA$. Furthermore, the crystal structure of I5PM is stabilized by one kind of $\mathrm{C}-\mathrm{H} \cdots \mathrm{N}$ bond $(2.239 \AA)$ between the two adjacent I5PM molecules in the same layer, and one kind of $\mathrm{C}-\mathrm{H} \cdots \mathrm{O}$ bond $(2.705 \AA)$ and five kinds of $\mathrm{C}-\mathrm{H} \cdots \pi$ bonds $(2.727-3.362 \AA)$ between the 1,4-dioxane solvent molecules and I5PM molecules

Table 2 Crystal data of I3PM, I3PMBn, I3PMBi, I4PMBn, I4PMBi, and I5PM

\begin{tabular}{|c|c|c|c|c|c|c|}
\hline & I3PM & I3PMBn & I3PMBi & I4PMBn & I4PMBi & I5PM \\
\hline Empirical formula & $\mathrm{C}_{38} \mathrm{H}_{38} \mathrm{~N}_{4} \mathrm{O}_{6}$ & $\mathrm{C}_{36} \mathrm{H}_{34} \mathrm{~N}_{4} \mathrm{O}$ & $\mathrm{C}_{36} \mathrm{H}_{34} \mathrm{~N}_{4} \mathrm{O}$ & $\mathrm{C}_{36} \mathrm{H}_{34} \mathrm{~N}_{4} \mathrm{O}$ & $\mathrm{C}_{36} \mathrm{H}_{34} \mathrm{~N}_{4} \mathrm{O}$ & $\mathrm{C}_{38} \mathrm{H}_{38} \mathrm{~N}_{4} \mathrm{O}_{6}$ \\
\hline Formula weight & 646.72 & 538.67 & 538.67 & 538.67 & 538.67 & 646.72 \\
\hline Temperature (K) & $298(2)$ & $293(2)$ & $296(2)$ & $296(2)$ & $273(2)$ & $298(2)$ \\
\hline Crystal system & Triclinic & Triclinic & Monoclinic & Monoclinic & Triclinic & Triclinic \\
\hline Space group & $P \overline{1}$ & $P \overline{1}$ & $P 12_{1} / c 1$ & $C 2 / c$ & $P \overline{1}$ & $P \overline{1}$ \\
\hline$Z$ & 2 & 2 & 4 & 8 & 4 & 2 \\
\hline$D_{\text {calcd }}\left[\mathrm{mg} / \mathrm{m}^{3}\right]$ & 1.231 & 1.187 & 0.893 & 1.159 & 1.019 & 1.234 \\
\hline$F(000)$ & 684 & 572 & 1144 & 2288 & 1144 & 684 \\
\hline$\theta$ range $\left[{ }^{\circ}\right]$ & $2.20-26.00$ & $1.76-25.00$ & $2.38-66.78$ & $2.31-65.00$ & $3.10-64.99$ & $2.32-24.92$ \\
\hline$R_{1}[I>2 \sigma(I)]$ & 0.0724 & 0.0832 & 0.0978 & 0.0793 & 0.0917 & 0.0811 \\
\hline $\mathrm{w} R_{2}[I>2 \sigma(I)]$ & 0.2047 & 0.1684 & 0.2622 & 0.2138 & 0.2407 & 0.2091 \\
\hline$a[\AA]$ & $10.4610(14)$ & $8.3257(12)$ & $19.030(3)$ & $39.856(3)$ & $11.5539(4)$ & $9.885(5)$ \\
\hline$b[\AA]$ & $12.7008(17)$ & $12.1141(17)$ & 21.991(3) & $8.1733(4)$ & $17.9477(6)$ & $13.056(6)$ \\
\hline$c[\AA]$ & $14.931(3)$ & $15.672(2)$ & $9.8034(12)$ & $19.7806(10)$ & $17.9799(5)$ & $15.400(8)$ \\
\hline$\alpha[\mathrm{deg}]$ & $101.290(3)$ & $100.531(3)$ & 90 & 90 & $105.002(2)$ & $70.424(8)$ \\
\hline$\beta[\mathrm{deg}]$ & $103.112(3)$ & $90.188(4)$ & $102.551(7)$ & $106.633(2)$ & $96.230(2)$ & $80.392(9)$ \\
\hline$\gamma[\mathrm{deg}]$ & $109.122(2)$ & $103.883(3)$ & 90 & 90 & $99.152(2)$ & $68.547(8)$ \\
\hline$V\left[\AA^{3}\right]$ & $1745.0(5)$ & $1506.8(4)$ & 4004.5(9) & $6174.0(6)$ & $3511.4(2)$ & $1740.7(15)$ \\
\hline GOF & 0.971 & 1.054 & 0.950 & 0.913 & 0.954 & 1.037 \\
\hline$R_{\text {(int) }}$ & 0.0300 & 0.0477 & 0.0837 & 0.1099 & 0.0896 & 0.0654 \\
\hline No. of reflcns collected & 13631 & 8415 & 22108 & 20863 & 40675 & 14608 \\
\hline No. of unique reflcns & 6740 & 5293 & 6829 & 5158 & 11555 & 6358 \\
\hline$R_{1}$ (all data) & 0.1338 & 0.1608 & 0.1414 & 0.1746 & 0.1375 & 0.1275 \\
\hline $\mathrm{w} R_{2}$ (all data) & 0.2363 & 0.2008 & 0.3153 & 0.2966 & 0.2779 & 0.2355 \\
\hline
\end{tabular}


Table 3 Summarization of the intermolecular interactions in I3PM, I3PMBn, I3PMBi, I4PMBn, I4PMBi, and I5PM

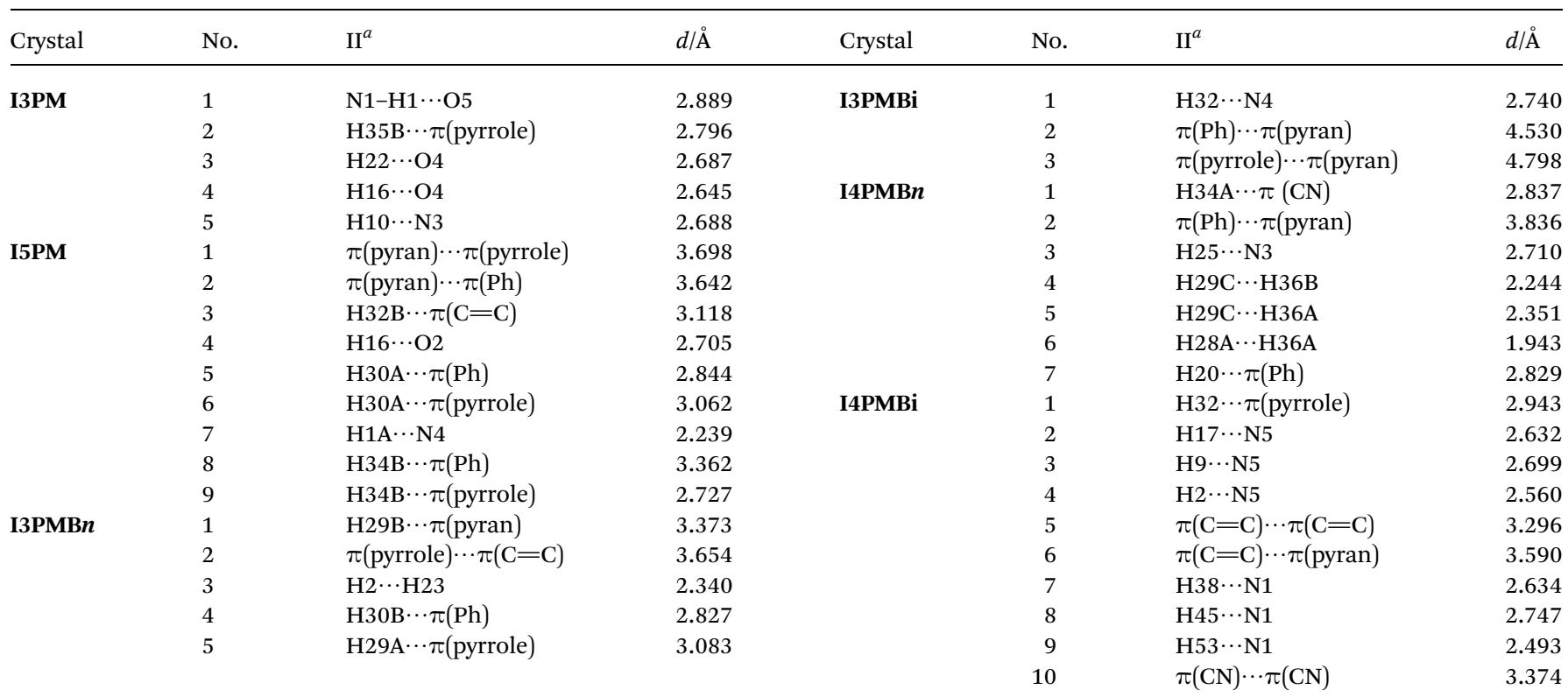

${ }^{a}$ Intermolecular interaction.

(Fig. 6 and Table 3). As a result, the molecules of I5PM pack in a reverse lamellar structure. For the crystals of I3PM and I5PM, the presence of solvent molecules indicates that there are a large number of defects or holes in their offset lamellar crystal structures. $^{23}$ This kind of loose crystal structure is easily destroyed by planarization of the molecular conformation or slip deformation upon grinding, which increases molecular conjugation and promotes a red shift of the emission spectra. ${ }^{23 a}$ The destruction of crystalline structures means the transformation from a crystalline state to an amorphous state, which is confirmed by the results of XRD experiments (Fig. 3 and S5 $\dagger$ ).
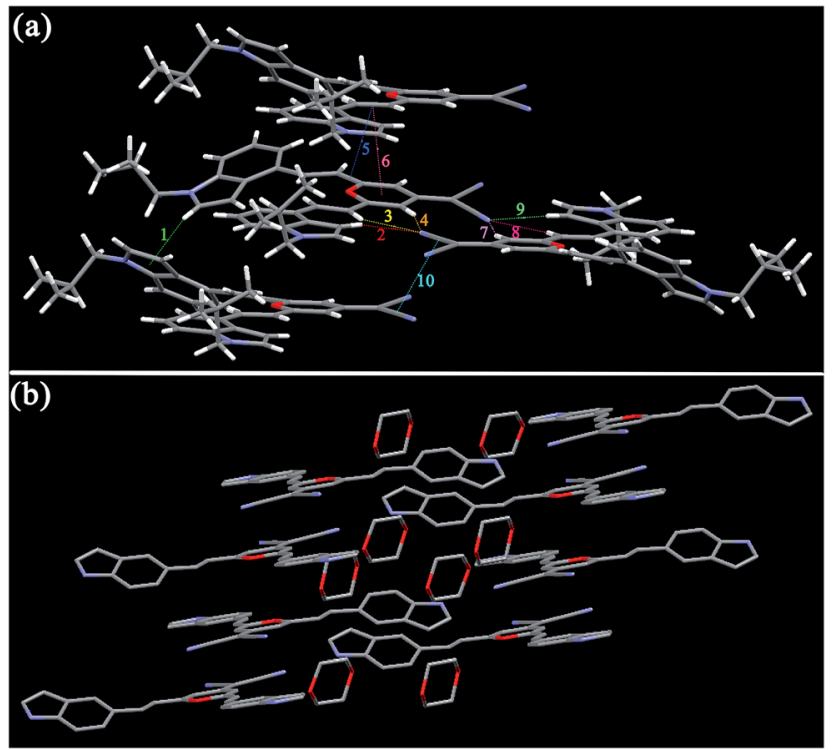

Fig. 6 Intermolecular interactions (a) and molecular packing (b) in I5PM.
In the case of crystals I3PMBn, I3PMBi, and I4PMBn, the two dihedral angles between the two indole units and the $4 H$-pyran cycle are 7.67 and $14.32^{\circ}$ (I3PMBn), 5.89 and $19.79^{\circ}$ (I3PMBi), and 10.01 and $13.04^{\circ}$ (I4PMBn), respectively. For crystal I4PMBi, there were two independent conformational molecules whose corresponding dihedral angles are 5.77 and $23.58^{\circ}$, and 5.23 and $30.22^{\circ}$, respectively. Based on the above mentioned, we can conclude the following results: (i) the introduction of $n$-butyl group and i-butyl group on the nitrogen atom of the indole unit enhances the degree of distortion of the molecules, which can be used to account for the obvious blue shift of the solid-state emission spectra of these four molecules relative to those of the corresponding parent molecules I3PM and I4PM (Table 1); (ii) compared with $n$-butyl group, i-butyl group is more favorable for the formation of a twisted conformation, and therefore the solid-state emissions of I3PMBi and I3PMBi show blue shift compared to those of I3PMBn and I3PMBn, respectively; (iii) I4PM derivatives possess a more twisted conformation than the corresponding I3PM derivatives, which is consistent with the fact that the former has shorter emission wavelength in the solid state.

Furthermore, we investigated the intermolecular interactions and packing arrangements of these compounds. For crystal I3PMBn, the distance between the indole unit and the olefinic double bond in the upper and lower molecules is measured to be $3.654 \AA$, indicating the existence of weak $\pi-\pi$ stacking (Fig. 7 and Table 3). Three kinds of aliphatic $\mathrm{C}-\mathrm{H} \cdots \pi$ bonds $(2.827,3.083$, and $3.373 \AA$ ), and one kind of van der Waals force between aromatic hydrogen atoms in the phenyl units with a distance of $2.340 \AA$ stabilizes the crystal structure. Similar to I3PM, I3PMBn adopts a reverse lamellar packing pattern. However, there is only one kind of $\mathrm{C}-\mathrm{H} \cdots \mathrm{N}$ bond $(2.740 \AA)$ in the crystal of I3PMBi, which results in 

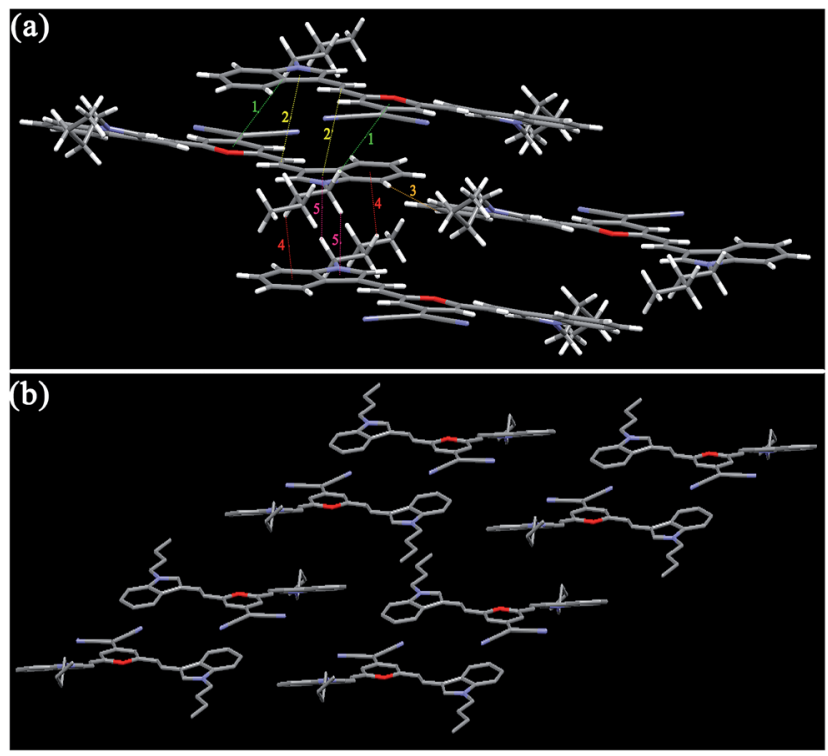

Fig. 7 Intermolecular interactions (a) and molecular packing (b) in I3PMBn.

a herringbone arrangement with a herringbone angle of $86.03^{\circ}$ (Fig. 8 and Table 3). Herein, the i-butyl group obviously decreases the intermolecular interactions between the molecules of I3PMBi, which causes that I3PMBi has larger $\Delta \lambda_{\text {MFC }}$ value and shows more significant solid-state fluorescence change compared with I3PMB $n$ containing $n$-butyl group.

As shown in Fig. 9, the molecules of I4PMB $n$ in the crystal are stabilized by one kind of $\pi-\pi$ stacking interaction (3.836 $\AA$ ) between the $4 H$-pyran ring and the phenyl unit, one kind of $\mathrm{C}-\mathrm{H} \cdots \mathrm{N}$ bond $(2.710 \AA)$, two kinds of $\mathrm{C}-\mathrm{H} \cdots \pi$ bonds $(2.829$ and $2.837 \AA$ ), and three kinds of van der Waals force between the hydrogen atoms in $n$-butyl groups (1.943, 2.244 and $2.351 \AA$ )
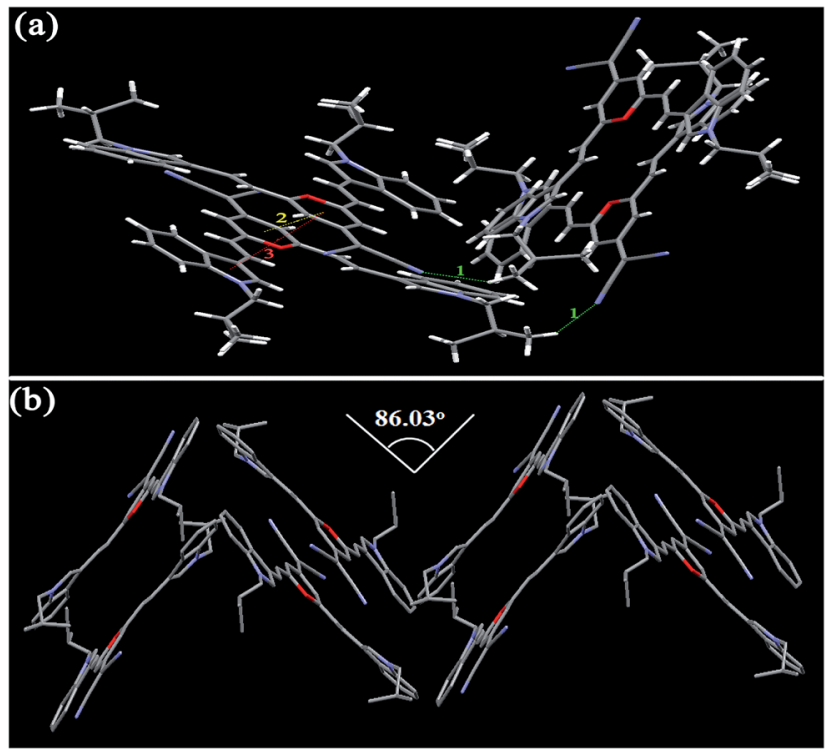

Fig. 8 Intermolecular interactions (a) and molecular packing (b) in I3PMBi.
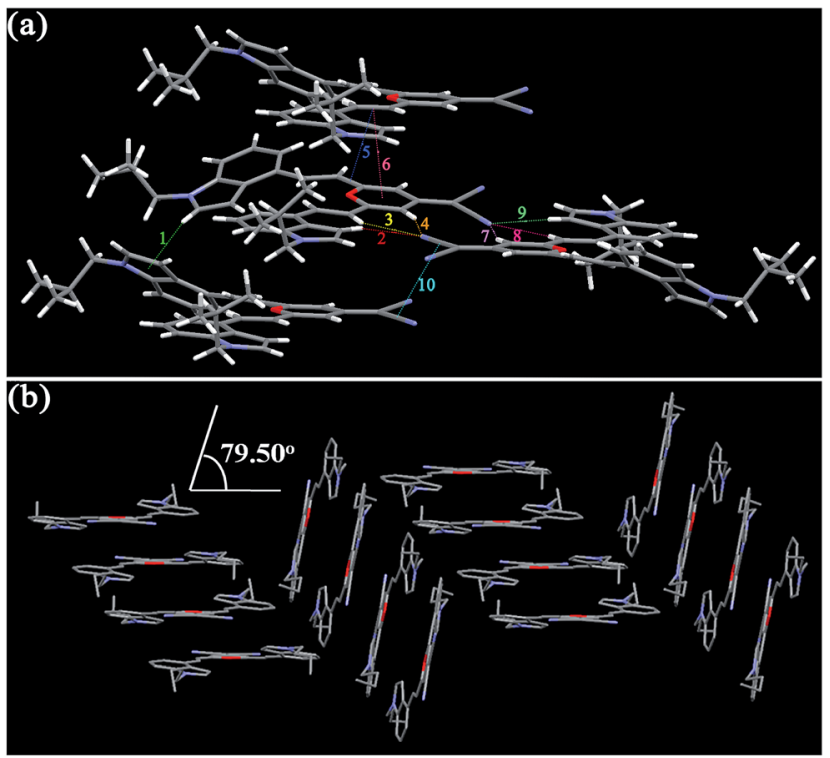

Fig. 9 Intermolecular interactions (a) and molecular packing (b) in I4PMBn.

(Table 3). The molecules of I4PMB $\boldsymbol{n}$ pack in a herringbone sharp including a lamellar arrangement and the herringbone angle is $79.50^{\circ}$, which is similar to those of I3PMBi. For crystal I4PMBi, six kinds of $\mathrm{C}-\mathrm{H} \cdots \mathrm{N}$ bonds $(2.493-2.747 \AA)$ restrict the molecules in the same layer, three kind of $\pi-\pi$ stacking interaction (3.296, 3.590 and $3.374 \AA$ ) and two kinds of $\mathrm{C}-\mathrm{H} \cdots \pi$ bonds (2.874 and $2.943 \AA$ ) stabilize the molecules in the upper and lower layers (Fig. 10 and Table 3). These intermolecular interactions cause the molecules of I4PMBi to adopt a face-to-face lamellar stacking mode. Herein, compared with the herringbone packing containing a lamellar arrangement of I4PMBn, purely lamellar arrangement of I4PMBi makes it easier to break down by external pressure, which leads to its larger $\Delta \lambda_{\mathrm{MFC}}$ value.

For the introduction of different alkyl chains, two factors, namely the isomerization of the alkyl chain and the alkyl chain length, are considered. In view of the I3PM and I4PM derivatives, according to the above crystal structure analysis, i-butyl group leads to a weaker intermolecular interaction and/or a looser stacking mode than $n$-butyl group due to a more twisted molecular conformation, which can be used to account for the fact that the isomerization of the alkyl chain is benefit for the remarkable MFC properties. But for the length of alkyl chain, its influence is obviously controlled by the substitution position of the indole unit according to the experimental results. The increase of the alkyl chain length is favorable to the MFC properties of the I3PM derivatives, whereas is unfavorable to those of the I4PM derivatives. This unusual phenomenon is likely to be related to the change of the molecular conformation before and after grinding. Because there is no strong $\pi-\pi$ stacking as well as other intermolecular interactions in the original crystalline samples of the I3PM and I4PM derivatives, their emissions should mainly depend on the molecular conformations. As shown in Table 1, the original crystalline samples of I3PMD and I4PMD show a blue shift and red shift 

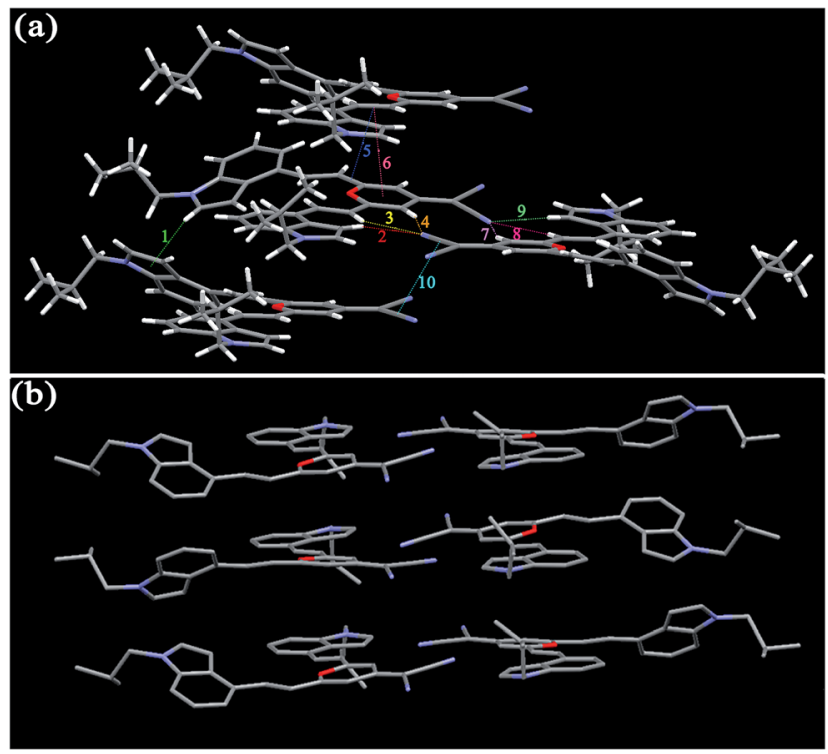

Fig. 10 Intermolecular interactions (a) and molecular packing (b) in I4PMBi.

compared with those of I3PMBn and I4PMBn, respectively, indicating I3PMD and I4PMBn might have a more twisted conformation and a poorer $\pi$-conjugation. After grinding, a better $\pi$-conjugation for the ground samples of I3PMD and I4PMB $\boldsymbol{n}$ is anticipated, and thus I3PMD and I4PMB $\boldsymbol{n}$ have a larger $\Delta \lambda_{\mathrm{MFC}}$ value. Based on the results in the case, a regular pattern can be concluded as follows: if the emission wavelengths of the original crystalline samples of a series of fluorescent compounds gradually blue-shifted with the increase of alkyl chain length, the homologue with longer alkyl chain will exhibit higher contrast MFC behavior after grinding; $;^{7-9,24}$ otherwise shorter alkyl chain is more beneficial to the MFC property, ${ }^{10-12}$ which is in good agreement with the previous reports.

\section{Conclusions}

A series of new fluorescent molecules based on dicyanomethylene- $4 H$-pyran and indole unit via alkene module were synthesized in order to investigate the combined effects of the introduction of different alkyl chains and the isomerization of indole unit on their MFC properties. The results show that the isomerization of indole unit plays a more important role in regulating their MFC activities than the choice of the alkyl chains. For the I3PM derivatives, only I3PMBi and I3PMD show obvious MFC phenomenon, and the increase of the alkyl chain length and the isomerization of the alkyl chain are favorable to their effective mechanofluorochromism. But interestingly, longer alkyl chain is not advantageous for the MFC activities of the I4PM derivatives. The opposite alkyl length-dependent mechanofluorochromism is closely related to the degree of distortion of the molecular conformation of the homologues, that is, the greater the degree of distortion of molecular conformation, the larger grinding-induced spectral shifts. More specially, the introduction of the alkyl chains is completely detrimental to those of the I5PM derivatives. Based on the results of crystal structure analysis, the weak intermolecular interactions and loose packing modes in MFC-active molecules result in that the crystalline structures of the molecules are easily destroyed by pressure stimulation and thus the morphological transformation of the molecular aggregates are realized. Although the transformation from a crystalline state to an amorphous state cannot fully determine whether a molecule has MFC properties, for example, I3PM and I3PMBn do not exhibit obvious solid-state fluorescence color change upon grinding, there is no doubt the transformation is crucial for the formation of MFC properties. This work provides a good design idea for designing and obtaining new MFC-active materials based on the traditional fluorescent molecular frameworks.

\section{Conflicts of interest}

There are no conflicts to declare.

\section{Acknowledgements}

This research work was supported by National Natural Science Foundation of China (Grants 21572165, 21472140, and 21372177) and Zhejiang Provincial Natural Science Foundation (Grant LY16B040005).

\section{Notes and references}

1 (a) S. J. Toal, K. A. Jones, D. Magde and W. C. Trogler, J. Am. Chem. Soc., 2005, 127, 11661-11665; (b) Z. Ning, Z. Chen, Q. Zhang, Y. Yan, S. Qian, Y. Cao and H. Tian, Adv. Funct. Mater., 2007, 17, 3799-3807; (c) B. Xu, M. Xie, J. He, B. Xu, Z. Chi, W. Tian, L. Jiang, F. Zhao, S. Liu, Y. Zhang, Z. Xu and J. Xu, Chem. Commun., 2013, 49, 273-275.

2 (a) S. Hirata and T. Watanabe, Adv. Mater., 2006, 18, 27252729; (b) S. J. Lim, B. K. An, S. D. Jung, M. A. Chung and S. Y. Park, Angew. Chem., Int. Ed., 2004, 43, 6346-6350; (c) K. Ariga, T. Mori and J. P. Hill, Adv. Mater., 2012, 24, 158-176.

3 (a) A. Kishimura, T. Yamashita, K. Yamaguchi and T. Aida, Nat. Mater., 2005, 4, 546-549; (b) H. Ito, T. Saito, N. Oshima, N. Kitamura, S. Ishizaka, Y. Hinatsu, M. Wakeshima, M. Kato, K. Tsuge and M. Sawamura, J. Am. Chem. Soc., 2008, 130, 10044-10045.

4 (a) W. Z. Yuan, Y. G. Gong, S. M. Chen, X. Y. Shen, J. W. Y. Lam, P. Lu, Y. W. Lu, Z. M. Wang, R. R. Hu, N. Xie, H. S. Kwok, Y. M. Zhang, J. Z. Sun and B. Z. Tang, Chem. Mater., 2012, 24, 1518-1528; (b) Y. Y. Gong, Y. R. Zhang, W. Z. Yuan, J. Z. Sun and Y. M. Zhang, J. Phys. Chem. C, 2014, 118, 10998-11005; (c) Y. Y. Gong, Y. Q. Tan, J. Liu, P. Lu, C. F. Feng, W. Z. Yuan, Y. W. Lu, J. Z. Sun, G. F. He and Y. M. Zhang, Chem. Commun., 2013, 49, 4009-4011.

5 Z. Chi, X. Zhang, B. Xu, X. Zhou, C. Ma, Y. Zhang, S. Liu and J. Xu, Chem. Soc. Rev., 2012, 41, 3878-3896.

6 S. Xue, X. Qiu, Q. Sun and W. Yang, J. Mater. Chem. C, 2016, 4, 1568-1578. 
7 L. Y. Bu, M. X. Sun, D. T. Zhang, W. Liu, Y. L. Wang, M. Zheng, S. F. Xue and W. J. Yang, J. Mater. Chem. C, 2013, 1, 2028-2035.

8 M. Zheng, M. X. Sun, Y. P. Li, J. F. Wang, L. Y. Bu, S. F. Xue and W. J. Yang, Dyes Pigm., 2014, 102, 29-34.

9 (a) X. Q. Zhang, Z. G. Chi, B. J. Xu, L. Jiang, X. Zhou, Y. Zhang, S. W. Liu and J. R. Xu, Chem. Commun., 2012, 48, 1089510897; (b) W. Liu, Y. L. Wang, M. X. Sun, D. T. Zhang, M. Zheng and W. J. Yang, Chem. Commun., 2013, 49, 60426044.

10 Y. L. Wang, W. Liu, L. Y. Bu, J. F. Li, M. Zheng, D. T. Zhang, M. X. Sun, Y. Tao, S. F. Xue and W. J. Yang, J. Mater. Chem. C, 2013, 1, 856-862.

11 Q. K. Sun, W. Liu, S. A. Ying, L. L. Wang, S. F. Xue and W. J. Yang, RSC Adv., 2015, 5, 73046-73050.

12 P. Xue, J. Sun, P. Chen, P. Gong, B. Yao, Z. Zhang, C. Qian and R. Lu, J. Mater. Chem. C, 2015, 3, 4086-4092.

13 Y. Xiong, X. Yan, Y. Ma, Y. Li, G. Yin and L. Chen, Chem. Commun., 2015, 51, 3403-3406.

14 (a) K. C. Naeem, A. Subhakumari, S. Varughese and V. C. Nair, J. Mater. Chem. C, 2015, 3, 10225-10231; (b) C. Niu, Y. You, L. Zhao, D. He, N. Na and J. Ouyang, Chem.-Eur. J., 2015, 21, 13983-13990.

15 (a) P. Rajamalli, P. Gandeepan, M. J. Huang and C. H. Cheng, J. Mater. Chem. C, 2015, 3, 3329-3335; (b) W. A. Morris, T. Liu and C. L. Fraser, J. Mater. Chem. C, 2015, 3, 352-363; (c) C. Ma, X. Zhang, Y. Yang, Z. Ma, L. Yang, Y. Wu, H. Liu, X. Jia and Y. Wei, Dyes Pigm., 2016, 129, 141-148.

16 (a) M. Yamaguchi, S. Ito, A. Hirose, K. Tanaka and Y. Chujo, J. Mater. Chem. C, 2016, 4, 5314-5319; (b) T. Jadhav, B. Dhokale, S. M. Mobin and R. Misra, RSC Adv., 2015, 5,
29878-29884; (c) W. Li, L. Wang, J. P. Zhang and H. Wang, J. Mater. Chem. C, 2014, 2, 1887-1892; (d) Y. J. Zhang, G. L. Zhuang, M. Ouyang, B. Hu, Q. B. Song, J. W. Sun, C. Zhang, C. Gu, Y. X. Xu and Y. G. Ma, Dyes Pigm., 2013, 98, 486-492; (e) Q. Song, Y. Wang, C. Hu, Y. Zhang, J. Sun, K. Wang and C. Zhang, New J. Chem., 2015, 39, 659-663.

17 Z. Guo, W. Zhu and H. Tian, Chem. Commun., 2012, 48, 60736084.

18 Y. Liu, Y. Lei, F. Li, J. Chen, M. Liu, X. Huang, W. Gao, H. Wu, J. Ding and Y. Cheng, J. Mater. Chem. C, 2016, 4, 2862-2870.

19 Y. Zhou, Y. Liu, Y. Guo, M. Liu, J. Chen, X. Huang, W. Gao, J. Ding, Y. Cheng and H. Wu, Dyes Pigm., 2017, 141, 428-440. 20 H. Li, Y. Guo, G. Li, H. Xiao, Y. Lei, X. Huang, J. Chen, H. Wu, J. Ding and Y. Cheng, J. Phys. Chem. C, 2015, 119, 6737-6748. 21 A. Meguellati, A. Ahmed-Belkacem, W. Yi, R. Haudecoeur, M. Crouillère, R. Brillet, J.-M. Pawlotsky, A. Boumendjel and M. Peuchmaur, Eur. J. Med. Chem., 2014, 80, 579-592.

22 CCDC 1554482-1554487† (I3PM, I3PMBn, I3PMBi, I4PMBn, I5PM, and I4PMBi).

23 (a) X. Zhou, H. Li, Z. Chi, X. Zhang, J. Zhang, B. Xu, Y. Zhang, S. Liu and J. Xu, New J. Chem., 2012, 36, 685-693; (b) J. Mei, J. Wang, A. Qin, H. Zhao, W. Yuan, Z. Zhao, H. H. Y. Sung, C. Deng, S. Zhang, I. D. Williams, J. Z. Sun and B. Z. Tang, J. Mater. Chem., 2012, 22, 4290-4298.

24 (a) X. Zhang, Z. Ma, Y. Yang, X. Zhang, Z. Chi, S. Liu, J. Xu, X. Jia and Y. Wei, Tetrahedron, 2014, 70, 924-929; (b) M. Zheng, D. T. Zhang, M. X. Sun, Y. P. Li, T. L. Liu, S. F. Xue and W. J. Yang, J. Mater. Chem. C, 2014, 2, 19131920; (c) W. Liu, Y. Wang, L. Bu, J. Li, M. Sun, D. Zhang, M. Zheng, C. Yang, S. Xue and W. Yang, J. Lumin., 2013, 143, 50-55. 